\title{
LARGE-EDDY SIMULATION OF THE STABLY STRATIFIED PLANETARY BOUNDARY LAYER
}

\author{
EILEEN M. SAIKI, CHIN-HOH MOENG and PETER P. SULLIVAN \\ National Center for Atmospheric Research, P.O. Box 3000, Boulder, Colorado 80307-3000, U.S.A.
}

(Received in final form 27 October 1999)

\begin{abstract}
In this work, we study the characteristics of a stably stratified atmospheric boundary layer using large-eddy simulation (LES). In order to simulate the stable planetary boundary layer, we developed a modified version of the two-part subgrid-scale model of Sullivan et al. This improved version of the model is used to simulate a highly cooled yet fairly windy stable boundary layer with a surface heat flux of $\langle w \theta\rangle_{o}=-0.05 \mathrm{~m} \mathrm{~K} \mathrm{~s}^{-1}$ and a geostrophic wind speed of $U_{g}=15 \mathrm{~m} \mathrm{~s}^{-1}$. Flow visualization and evaluation of the turbulence statistics from this case reveal the development of a continuously turbulent boundary layer with small-scale structures. The stability of the boundary layer coupled with the presence of a strong capping inversion results in the development of a dominant gravity wave at the top of the stable boundary layer that appears to be related to the most unstable wave predicted by the Taylor-Goldstein equation. As a result of the decay of turbulence aloft, a strong-low level jet forms above the boundary layer. The time dependent behaviour of the jet is compared with Blackadar's inertial oscillation analysis.
\end{abstract}

Keywords: Large-eddy simulation, Stable boundary layer, Gravity waves, Nocturnal jet.

\section{Introduction}

While the convective atmospheric boundary layer has historically been studied in great detail, its nocturnal counterpart, the stable boundary layer (SBL), has been somewhat neglected. This is due mainly to the difficulties associated with examining the stable boundary layer observationally, theoretically, and computationally as the stable boundary layer can range from being fully turbulent to highly intermittent depending upon the counteracting forces of buoyancy damping and shear production. The SBL is also complicated by additional characteristics such as sensitivity to sloping terrain, the presence of low-level jets, and the development of gravity waves (Stull, 1988; Mahrt, 1998). All of these factors contribute to the unsteady and variable nature of the SBL.

However, these complications do not outweigh the importance of studying and understanding the SBL. In addition to being of fundamental interest, SBL turbulence can have a day to day impact on our lives. For example, the effects of stability lead to a decrease in turbulent vertical mixing that under normal circumstances allows dispersion of contaminants injected into the atmosphere. This behaviour can lead to the development of horizontal layering of pollutants, which can build up over time if the boundary layer is very stable. 
Numerical simulations have been one of the key components used to study the atmospheric boundary layer. In particular, large-eddy simulation (LES) has been successful in studying convective and neutrally stratified boundary layers (Moeng, 1984; Moeng and Sullivan, 1994; Sullivan et al., 1994, 1996). In LES, only the large-scale structures resolved by the computational grid are directly simulated while the effects of the small scales that fall below the grid resolution are parameterized by a subgrid-scale (SGS) model. More recently, LES has also been applied in a number of studies investigating the stable boundary layer.

One of the first LES of the SBL was performed by Mason and Derbyshire (1990) using a Smagorinsky SGS-based model. This original attempt was important in demonstrating the feasibility of using LES to simulate the weakly stable boundary layer. In their work, typical turbulent statistics and profiles were examined and comparisons were made with the second-order closure model of Brost and Wyngaard (1978), the gradient closure models of Hunt (1985) and the local scaling theory of Nieuwstadt (1984). The LES results were used by Derbyshire (1990) to further support and extend the theory of Nieuwstadt (1984), and comparisons of the results with field measurements of the SBL at several observational sites were discussed in Derbyshire (1995). Follow up studies to this earlier work improved the surface-layer profiles of velocity and temperature by adding stochastic backscatter to the subgrid-scale model (Brown et al., 1994).

The weakly stratified boundary layer with and without a weak capping inversion imposed above the boundary layer was studied by Andren (1995). In his study, comparisons between LES using the original SGS model developed in Moeng (1984) and the later modified version of Sullivan et al. (1994) were made, revealing that the modified model produced better agreement of the temperature and mean velocity profiles with Monin-Obukhov similarity relations in the surface layer. The presence of a weak capping inversion led to the development of wave motions above the boundary layer whose overall effect on the SBL was small. The LES results were used to characterize the SBL in terms of local similarity, turbulence budgets, and quadrant analysis. Recently, the differences and similarities between different numerical models simulating the SBL were highlighted in a study by Galmarini et al. (1998), where the profiles of various quantities such as variances, fluxes, and nondimensional gradients of temperature and velocity from Andren (1995) were contrasted with those obtained from a LES using a different SGS model, and from a one-dimensional second-order closure model.

Kosovic and Curry (2000) implemented a newly developed SGS model to perform a LES of a quasi-steady state SBL in the Arctic circle area (latitude: $\phi=73^{\circ}$ ) where the SBL can persist for long periods of time. The computation was performed over 24 hours of physical time, which revealed a quasi-steady state of the SBL after about 10 hours. During this time, a low-level maximum in the mean wind profile associated with a damped inertial oscillation and the development of an elevated inversion were observed. A number of computations were performed comparing the effects of varying parameters such as geostrophic wind speed, cool- 
ing rate, and latitude. From these simulations, theoretical formulae for predicting the boundary-layer height and models for the SBL were tested.

The SBL problem has also been studied using direct numerical simulation (DNS) of a stable Ekman layer at low Reynolds number (Coleman et al., 1992). In their study, turbulent statistics were evaluated, and closure models and local scaling theories were tested. They observed general agreement with some of the LES results of Mason and Derbyshire (1990). In particular, their results showed a tendency to support the local scaling theory of Nieuwstadt (1984) and gradient closure models of Hunt et al. (1995). However, other comparisons with closure models such as Brost and Wyngaard (1978) showed good agreement only when the data were adjusted for low Reynolds number effects.

One of the difficulties associated with LES of the SBL is that, as stability increases, the dominant eddies become smaller. This places the burden of simulating the flow field on the SGS model and, consequently, the results can be sensitive to the type of parameterization used to represent the effects of the subgrid scales. Thus, the majority of previous studies have focused on modelling weakly stable boundary layers, i.e., boundary layers with continuous turbulence and little intermittency (Mahrt, 1998). This involves applying a small cooling rate, $\left(\frac{\partial\langle\theta\rangle}{\partial t}\right)_{o}$, or small negative heat flux, $\langle w \theta\rangle_{o}$, at the surface and/or imposing a high geostrophic velocity, $U_{g}$. This problem can be partially alleviated by using computational meshes with higher resolution than those used for convective or neutral boundary layers, and as a result smaller computational domain sizes have been typically used in previous LES studies.

An original goal of this work was to test the limits of the two part SGS model of Sullivan et al. (1994) to simulate a very stable boundary layer that is highly intermittent. The objective was to ultimately simulate the layering or fanning effect (Stull, 1988) that can develop in the SBL, causing pollutants to spread out horizontally. Furthermore, we were interested in studying the formation of the lowlevel jet, which in the very stable boundary layer may produce turbulence aloft that is detached from the surface (Mahrt, 1998). However, we found that the first goal was unattainable using the existing SGS model and a revision of the model was required. Using this modified SGS model (Section 2), we simulate a SBL that is continuously turbulent with a strong geostrophic wind $\left(U_{g}=15 \mathrm{~m} \mathrm{~s}^{-1}\right)$ and stability that is weak-to-moderate; the stability index characterizing the SBL is $\frac{h}{L}=2.3$, where $h$ is the SBL height and $L$ is the Obukhov length. For this SBL, we examine some of the turbulent statistics of the flow and perform detailed flow visualization and quadrant analysis. In addition, we examine the development of a strong lowlevel jet that develops at the top of the SBL and compare its development with the predictions from Blackadar's (1957) model. 


\section{Procedure}

The LES code originally outlined in Moeng (1984) and modified later in Sullivan et al. $(1994,1996)$ is implemented in this study. As noted in the introduction and detailed in the next section, the SGS model was further modified for the highly cooled SBL. The LES equations are integrated in time using an explicit multistage third-order Runge-Kutta scheme. Spatially, the equations are discretized by a pseudo-spectral method with Fourier transforms in the homogeneous directions, $x$ and $y$, and second-order finite differences in the vertical direction $(z)$. The boundary conditions at the surface are constructed using Monin-Obukhov (MO) similarity theory, while a radiation condition (Klemp and Durran, 1983) is applied at the upper boundary in order to allow gravity waves to propagate out of the computational domain without reflection.

\subsection{SUbGRID-SCALE MODEL}

Preliminary computations of highly cooled SBLs using the original two part SGS model developed by Sullivan et al. (1994) led to the development of unphysical profiles in various turbulent quantities, which occurred due to the collapse of the SGS vertical heat flux, $\tau_{\theta 3}$, to zero near the surface. This behaviour occurred contrary to the assumptions associated with LES in the atmosphere where it is expected that the majority of turbulent motions near the surface are subgrid-scale such that the SGS fluxes cannot fall to zero there. This assumption can be observed by examining the total horizontally averaged vertical heat flux as a function of height $(z)$ for LES, which is composed of the resolved flux, $w \theta$, and the subgrid-scale flux, $\tau_{\theta 3}$, i.e.,

$$
(w \theta)_{\text {total }}(z)=w \theta(z)+\tau_{\theta 3}(z) .
$$

At the surface $(z=0 \mathrm{~m})$, a boundary condition in the form of a horizontally averaged negative heat flux, $\langle w \theta\rangle_{o}$, is applied in order to cool the boundary layer such that $(w \theta)_{\text {total }}(z=0)=\langle w \theta\rangle_{o}$. The boundary conditions on vertical velocity, $w$, at the surface further requires that $w \theta(z=0)$ decreases to zero, leaving $\tau_{\theta_{3}}$ to represent the bulk of the heat flux near the ground. Note that at the lowest grid point where $z=0 \mathrm{~m}, \tau_{\theta 3}$ is imposed directly using the boundary condition defined in Moeng (1984), while $\tau_{\theta 3}$ at the grid points above $z=0 \mathrm{~m}$ is determined by the SGS model outlined below.

The observed decrease in $\tau_{\theta 3}$ near the surface in our earlier computations can be attributed to the SGS model, which relates $\tau_{\theta 3}$ to the SGS kinetic energy, $e$, and the length scale, $l$,

$$
\tau_{\theta 3}=-v_{\theta} \frac{\partial \theta}{\partial z}
$$

where

$$
v_{\theta}=\left(1+\frac{2 l}{\Delta}\right) v_{t},
$$


and

$$
v_{t}=C_{k} l e^{\frac{1}{2}} .
$$

The SGS energy, $e$ is determined using the equation

$$
\frac{\partial e}{\partial t}+u_{j} \frac{\partial e}{\partial x_{j}}=-\tau_{i j} S_{i j}+\frac{g}{\theta_{o}} \tau_{\theta 3}-\epsilon+\frac{\partial}{\partial x_{i}}\left(2 v_{t} \frac{\partial e}{\partial x_{i}}\right) \text {, }
$$

where the SGS dissipation, $\epsilon$, is

$$
\epsilon=C_{\epsilon} \frac{e^{\frac{3}{2}}}{l}
$$

The coefficients in the above equations are specified as $C_{\epsilon}=\left(0.19+0.74 \frac{l}{\Delta}\right)$ and $C_{k}=0.1$, where $\Delta$ is an average grid spacing. The length scale is defined as either $l=\Delta$ for neutral or convectively unstable regions, or as

$$
l=0.76\left(\frac{e^{\frac{1}{2}}}{N}\right)
$$

for stably stratified regions (Deardorff, 1980; Moeng, 1984). Here, $N$ is the BruntVäisälä frequency defined by $N^{2}=\frac{g}{\theta_{o}} \frac{\partial\langle\theta\rangle}{\partial z}$, where $\frac{g}{\theta_{o}}$ is the buoyancy coefficient. From the above relations, it can be observed that with increasing stratification, $N$ increases (especially near the surface where the boundary layer is very stable) and $l$ will decrease. In addition, the SGS energy for the SBL will decrease with increasing stability as the buoyancy destruction term in Equation (5) increases relative to the shear term. With both $e$ and $l$ falling to zero near the surface, the eddy coefficients in Equations 3 and 4 decrease to zero and as a result the SGS heat flux (Equation 2) becomes zero as well. Although this model was used in the work of Andren (1995), the above behaviour was not observed as the boundary layer was only weakly cooled with a small surface flux of $\langle w \theta\rangle_{o}=-0.005 \mathrm{~m} \mathrm{~K} \mathrm{~s}^{-1}$.

Note that, although the SGS momentum flux, $\tau_{i j}$, depends on $l$ and $e$ in a similar manner, the two part nature of the model for $\tau_{i j}$ does not allow it to fall to zero near the surface. From Sullivan et al. (1994),

$$
\tau_{i j}=-2 v_{t} \gamma S_{i j}-2 v_{T}\left\langle S_{i j}\right\rangle
$$

where $S_{i j}$ is the rate of strain tensor,

$$
S_{i j}=\left(\frac{\partial u_{i}}{\partial x_{j}}+\frac{\partial u_{j}}{\partial x_{i}}\right)
$$

$v_{t}$ is defined as in Equation (4), and the mean eddy viscosity, $v_{T}$, is 


$$
\begin{aligned}
v_{T} & =v_{T}^{*} \frac{k z_{1}}{u_{*} \phi_{m}\left(z_{1}\right)} \sqrt{2\left\langle S_{i j} S_{i j}\right\rangle} \text { if } \mathrm{z} \neq \mathrm{z}_{1} \\
& =v_{T}^{*} \text { if } \mathrm{z}=\mathrm{z}_{1} .
\end{aligned}
$$

In these equations, $k$ is the von Kármán constant, $u_{*}$ is the friction velocity, $z_{1}$ is the location of the first grid point above the surface, $\phi_{m}(z)$ is the M-O stability function for velocity (Businger et al., 1971),

$$
\frac{d U}{d z}=\frac{u_{*} \phi_{m}(z)}{k z}
$$

and the near surface mean eddy viscosity is

$$
v_{T}^{*}=\frac{u_{*} k z_{1}}{\phi_{m}\left(z_{1}\right)}-\left\langle v_{t} \gamma\right\rangle-\frac{k z_{1}}{u_{*} \phi_{m}\left(z_{1}\right)}\left[\langle u w\rangle_{z=z_{1}}^{2}+\langle v w\rangle_{z=z_{1}}^{2}\right]^{\frac{1}{2}} .
$$

The isotropy factor, $\gamma$, is used to account for anisotropic effects near the surface and to shift the SGS model from a LES type of parameterization for the subgrid-scales, to an ensemble average type that models all small scales. In the above relations, \langle\rangle denotes horizontal averages. This two part model was developed in order to improve the velocity and temperature profiles in the surface layer in comparison with Monin-Obukhov similarity forms.

As noted above, the form of the model in Equation (8) keeps $\tau_{i j}$ from going to zero near the surface. This feature led us to consider applying a similar two part model to represent $\tau_{\theta 3}$, i.e.,

$$
\tau_{\theta 3}=-v_{\theta} \gamma \frac{\partial \theta}{\partial z}-v_{\Theta} \frac{\partial\langle\theta\rangle}{\partial z} .
$$

The mean eddy diffusivity, $v_{\Theta}$, is determined by matching the computed temperature derivatives at the first grid point, $z_{1}=\Delta z$, with the Monin-Obukhov similarity forms. The $\mathrm{M}-\mathrm{O}$ relationship for temperature is (Businger et al., 1971)

$$
\frac{d\langle\theta\rangle}{d z}=\frac{\theta_{*} \phi_{h}(z)}{k z}
$$

where $\phi_{h}(z)$ is the $\mathrm{M}-\mathrm{O}$ stability function for temperature and $\theta_{*}=\frac{-\langle w \theta\rangle_{o}}{u_{*}}$ is the surface-layer temperature scale.

First, a Prandtl number relationship between $v_{\Theta}$ and the mean eddy viscosity, $v_{T}$, is assumed,

$$
v_{\Theta}=c_{\theta} v_{T}
$$


where $c_{\theta}$ can be considered to be a 'mean turbulent' Prandtl number. Next, a 'constant flux' condition below $z=z_{1}$ is imposed such that the sum of the resolved and SGS heat fluxes equals the surface heat flux, and from Equation (1) we obtain

$$
\langle w \theta\rangle_{o}=\langle w \theta\rangle_{z=z_{1}}+\left\langle\tau_{\theta 3}\right\rangle_{z=z_{1}} .
$$

Next, we approximate the horizontal average of Equation (13) at $z=z_{1}$ as,

$$
\left\langle\tau_{\theta 3}\right\rangle_{z=z_{1}}=-\left.\left(\left\langle v_{\theta} \gamma\right\rangle+v_{\Theta}\right) \frac{\partial\langle\theta\rangle}{\partial z}\right|_{z=z_{1}}
$$

by neglecting the fluctuating component of temperature in comparison with the mean temperature. The substitution of Equation (17) and Equation (14) into Equation (16) results in an expression for the near surface mean-field eddy diffusivity, $v_{\Theta}^{*}$, at $z=z_{1}$, i.e.,

$$
v_{\Theta}^{*}=\frac{k z_{1}}{\theta_{*} \phi_{h}\left(z_{1}\right)}\left(\langle w \theta\rangle_{z=z_{1}}-\langle w \theta\rangle_{o}\right)-\left\langle v_{\theta} \gamma\right\rangle .
$$

The coefficient $v_{\Theta}$ can then be found at all other levels where $z \neq z_{1}$ using Equation (15),

$$
v_{\Theta}=\frac{v_{\Theta}^{*}}{v_{T}^{*}} v_{T},
$$

such that the 'mean turbulent' Prandtl number is

$$
c_{\theta}=\frac{v_{\Theta}^{*}}{v_{T}^{*}} .
$$

As will be shown later, this two part model for $\tau_{\theta 3}$ successfully prevents the SGS part of the vertical heat flux from falling to zero near the surface.

\subsection{CONDitions OF THE COMPUTATION}

The computations were performed on a one kilometre square domain with $96 \times$ $96 \times 96$ evenly spaced grid points. The initial conditions consisted of a convective boundary layer (CBL) with a geostrophic wind of $\left(U_{g}, V_{g}\right)=(15,0) \mathrm{m} \mathrm{s}^{-1}$ driven by a positive heat flux of $\langle w \theta\rangle_{o}=0.05 \mathrm{~m} \mathrm{~K} \mathrm{~s}^{-1}$ applied at the surface. The surface roughness was $z_{o}=0.1 \mathrm{~m}$. The initial mean potential temperature profile imposed a steep capping inversion at $z=500 \mathrm{~m}$ where the temperature increased $8 \mathrm{~K}$ over twelve $\Delta z$ levels $\left(0.06 \mathrm{~K} \mathrm{~m}^{-1}\right)$. Above the capping inversion the temperature increased at a lapse rate of $0.003 \mathrm{~K} \mathrm{~m}^{-1}$. The capping inversion was included to limit the growth of the boundary layer. Initially, there was no strong capping inversion and the turbulence in the boundary layer grew to a height of $z=$ 
$800 \mathrm{~m}$ near the top of the domain. As a result, when the boundary layer was cooled, a nocturnal jet formed at this high level. Thus, the capping inversion was imposed to 'contain' the turbulence and bring the jet down to a level closer to the surface. The inversion is also physically based since after strong convection during the day a strong overlying inversion will develop.

After an hour of physical time, the heat flux was set to zero and a neutral boundary layer was simulated for two hours. This was followed by cooling the boundary layer with a negative heat flux. Besides problems associated with the SGS model itself (which were remedied by the two part SGS model), other difficulties arose when we attempted to cool the boundary layer too quickly, i.e., suddenly applied a large negative temperature flux $\left(\langle w \theta\rangle_{o}=-0.05 \mathrm{~m} \mathrm{~K} \mathrm{~s}^{-1}\right)$ at the ground to the neutral boundary layer. The vertical subgrid stress and temperature flux profiles would develop kinks at the second grid point above the ground. This behaviour was avoided by applying a monotone scheme for discretizing the vertical temperature advection terms and by gradually cooling the boundary layer to its highest level, $\langle w \theta\rangle_{o}=-0.05 \mathrm{~m} \mathrm{~K} \mathrm{~s}^{-1}$, over a time period of six hours starting from $\langle w \theta\rangle_{o}=-0.02 \mathrm{~m} \mathrm{~K} \mathrm{~s}^{-1}$.

A theoretical expression placing limits on the maximum heat flux that can be sustained in a stable boundary layer was derived by Derbyshire (1990)

$$
\left(\langle w \theta\rangle_{o}\right)_{\max }=\frac{\theta_{o} R_{f}}{g \sqrt{3}} U_{g}^{2}|f|,
$$

where $R_{f}=0.25$ is the critical flux Richardson number, $f$ is the Coriolis parameter, $g$ is the gravitational acceleration, and || denotes the absolute value. This expression with $\theta_{o}=300 \mathrm{~K}$ and $U_{g}=15 \mathrm{~m} \mathrm{~s}^{-1}$ gives a maximum absolute value of $\left(\langle w \theta\rangle_{o}\right)_{\max }=0.1 \mathrm{~m} \mathrm{~K} \mathrm{~s}^{-1}$, which lies well above the highest heat flux $\left(\left|\langle w \theta\rangle_{o}\right|=0.05 \mathrm{~m} \mathrm{~K} \mathrm{~s}^{-1}\right)$ applied in the current simulation. Based upon the fact that the conditions in our simulations do not exceed this theoretical limit, we would expect to be able to simulate a stable boundary layer that can support the cooling heat flux imposed at the surface.

After this period of gradual cooling, the stable boundary layer was then cooled further at $\langle w \theta\rangle_{o}=-0.05 \mathrm{~m} \mathrm{~K} \mathrm{~s}^{-1}$ for another five hours. During this period of time the boundary layer appeared to reach a quasi-steady state despite the high cooling flux, as the boundary layer was fairly windy allowing for continuous production of turbulence. The total amount of time that the boundary layer was cooled was about eleven hours at a latitude of $\phi=45^{\circ}$. Data at eighty second intervals were saved over the last 1.25 hours of the computation equivalent to nearly five eddy turnover times, $\tau=\frac{h}{u_{*}} \approx 880 \mathrm{~s}$. Here, $h$ is the height of the boundary layer based upon an estimate of the location where the horizontal stresses decreased to zero. It should be noted that, in essence, this case is the same as the neutral case studied in Moeng and Sullivan (1994) with the exception that the boundary layer is cooled and hence stably stratified. 
TABLE I

Bulk parameters. $u_{*}$ is the shear velocity, $L$ is the Obukhov length at the surface, $h$ is the height of the boundary layer, and $\alpha$ is the ageostrophic angle.

\begin{tabular}{lllll}
\hline$u_{*}\left(\mathrm{~m} \mathrm{~s}^{-1}\right)$ & $L(\mathrm{~m})$ & $h(\mathrm{~m})$ & $\frac{h}{L}$ & $\alpha(\mathrm{deg})$ \\
\hline 0.5 & 193.4 & 442.5 & 2.3 & 31.1 \\
\hline
\end{tabular}

\section{Results}

\subsection{BULK QUANTITIES}

The bulk parameters typically examined in boundary layer studies are presented in Table I. For the stability ratio, $\frac{h}{L}$, the Obukhov length at the surface is defined as $L=-\frac{u_{*}^{3}}{k \frac{g}{\theta_{o}}\langle w \theta\rangle_{o}}$. In comparison with previous LES of the SBL, both $u_{*}$, and $h$ obtained from the current simulation are higher, however this is due in part to the higher geostrophic velocity $\left(U_{g}=15 \mathrm{~m} \mathrm{~s}^{-1}\right)$ applied in the current simulations. The gradual cooling of the boundary layer aids in preventing a sudden collapse of the turbulence such that turbulence is produced continuously even during the period when the highest cooling heat flux is applied. We found that in cases where large cooling fluxes were suddenly imposed, there was a dramatic reduction in turbulence production aloft and hence in the height of the boundary layer. The height of the boundary layer can also be influenced by the low-level jet which develops above the SBL as the shear on the underside of the jet is a source for turbulence production. This issue will be discussed further in a subsequent section.

The combined effect of the applied geostrophic velocity and cooling flux results in a stability parameter, $\frac{h}{L}$, of 2.3 , which is higher than the cases investigated by Andren (1995) and falls in the middle of the cases examined by Brown (1994) and Kosovic and Curry (2000). The ageostrophic angle, $\alpha$, also falls within the values measured from these earlier LES studies.

\subsection{FIRST-ORDER STATISTICS}

The effect of the two part SGS model can be observed in Figure 1 where horizontally averaged total, SGS, and resolved vertical heat flux profiles, time averaged over the last 1.25 hours of the simulation, are presented. The SGS part does not decrease to zero near the surface, but increases smoothly towards the prescribed surface heat flux value of $\langle w \theta\rangle_{o}=-0.05 \mathrm{~m} \mathrm{~K} \mathrm{~s}^{-1}$ in accordance with the design of Equation (13). The total heat flux profile is nearly linear indicating that the flow field has reached a quasi-steady state (although the evolution of various quantities 


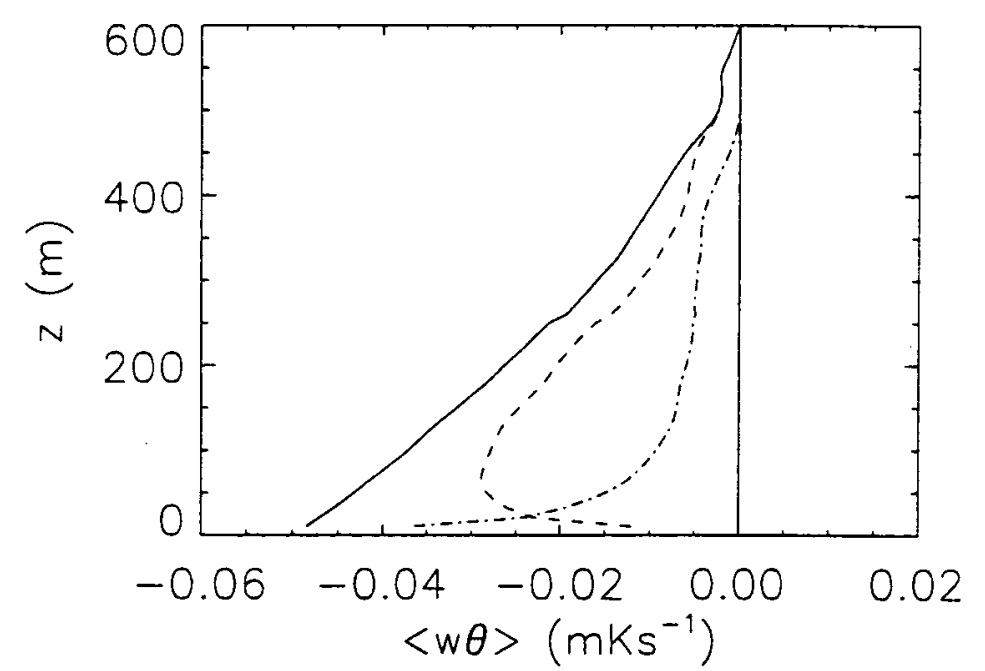

Figure 1. Vertical profiles of heat flux, $\langle w \theta\rangle$. Dashed line, resolved; dashed-dotted line, subgrid; solid line, total. In this and the following figures, all vertical profiles have been horizontally averaged at each level over the whole computational domain and time averaged over the last 1.25 hours of the simulation.

related to the low-level jet are still varying slowly in time, Figures 12 and 13). The strong capping inversion imposed in the initial condition is still present near $z=$ $500 \mathrm{~m}$, and consequently, as shown in the next section, gravity waves develop at this level. In addition, the height where the heat flux goes to zero is higher than the level where the momentum fluxes reach zero. This feature is the main reason why we chose to define the height of the boundary layer based upon an estimation of the location where the horizontal shear stresses would fall to zero. Following Kosovic and Curry (2000), we determine the height where the horizontal shear stress falls to $5 \%$ of the surface value and linearly extrapolate to the level where the stress would be zero assuming a linear stress profile. In reality, the horizontal stress profiles follow the $\frac{3}{2}$ power law as predicted by the theory for stationary stable boundary layers (Nieuwstadt, 1984), however the assumption of a linear stress profile near the top of the boundary layer to determine the height of the boundary layer gives a reasonable estimate of the top of the boundary layer. This can be observed in the $\langle u w\rangle$ and $\langle v w\rangle$ profiles in Figures $2 \mathrm{a}, \mathrm{b}$ where the curves reach zero near the estimated value of $h=442.5 \mathrm{~m}$.

Distributions of the gradient $\left(\mathrm{Ri}_{g}\right)$ and flux $\left(\mathrm{Ri}_{f}\right)$ Richardson numbers computed from horizontally averaged velocity and temperature reveal the relative effects of shear and buoyancy on the boundary layer in Figure 3. The gradient Richardson number increases upward from the surface until $z \approx 200 \mathrm{~m}$, at which point it remains fairly constant at $\mathrm{Ri}_{g}=0.2$ until near the top of the boundary layer where it reaches the critical Richardson number, $\left(\mathrm{Ri}_{g}\right)_{c}=0.25$, at $z=h=442.5 \mathrm{~m}$. It continues to increase above the SBL due to the effect of the large temperature 

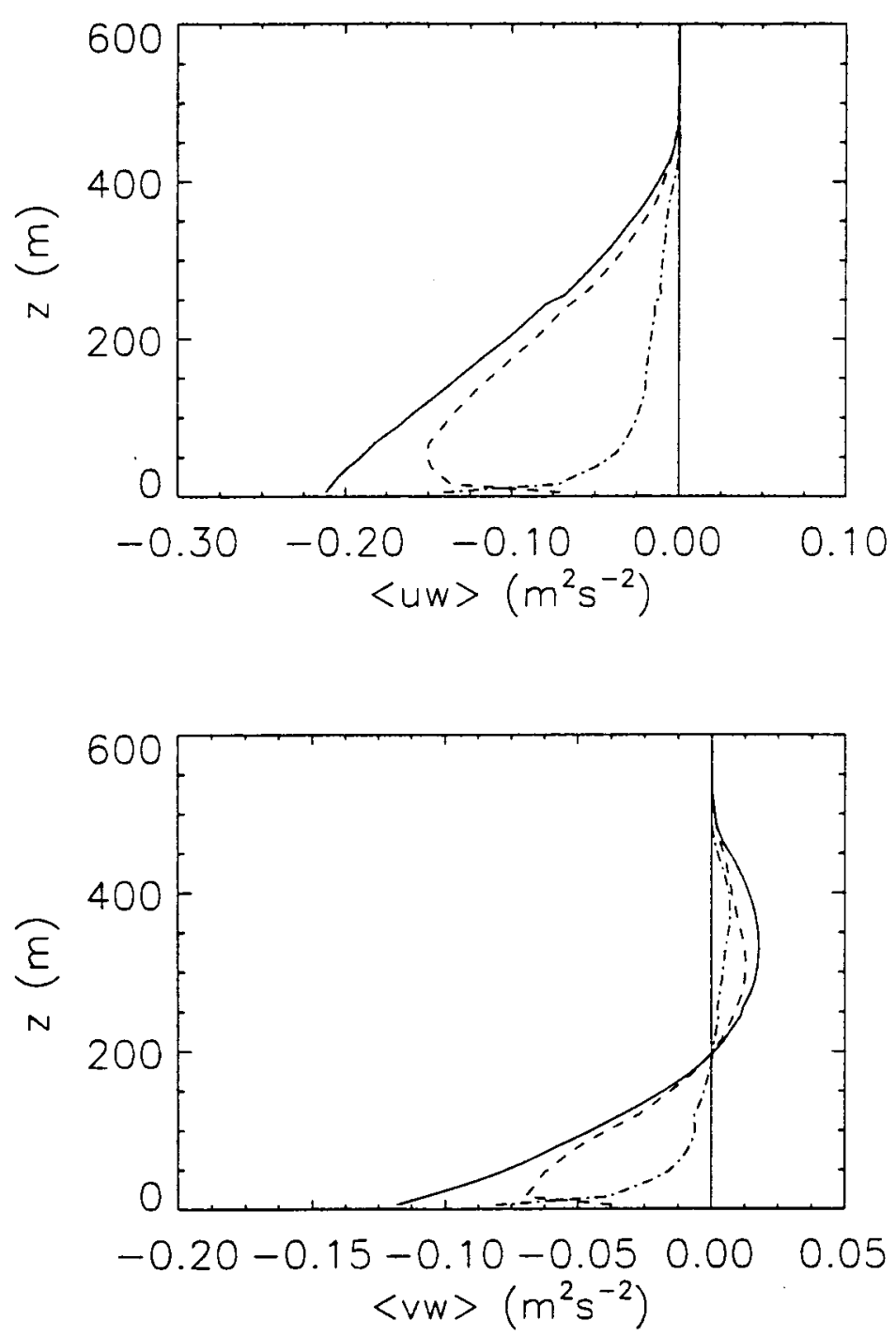

Figure 2. Vertical profiles of horizontal stresses, $\langle u w\rangle$ and $\langle v w\rangle$. Dashed line, resolved; dashed-dotted line, subgrid; solid line, total.

gradient imposed by the strong inversion interacting with the velocity gradient, $\frac{\partial U}{\partial z}$, associated with the upper side of the low-level jet.

The flux Richardson number is slightly higher than $\mathrm{Ri}_{g}$ throughout the boundary layer as it continuously increases all the way up to the top of the SBL. The definition of the flux Richardson number

$$
\operatorname{Ri}_{f}=\frac{\frac{g}{\theta_{o}}\langle w \theta\rangle}{\langle u w\rangle \frac{\partial U}{\partial z}+\langle v w\rangle \frac{\partial V}{\partial z}}
$$




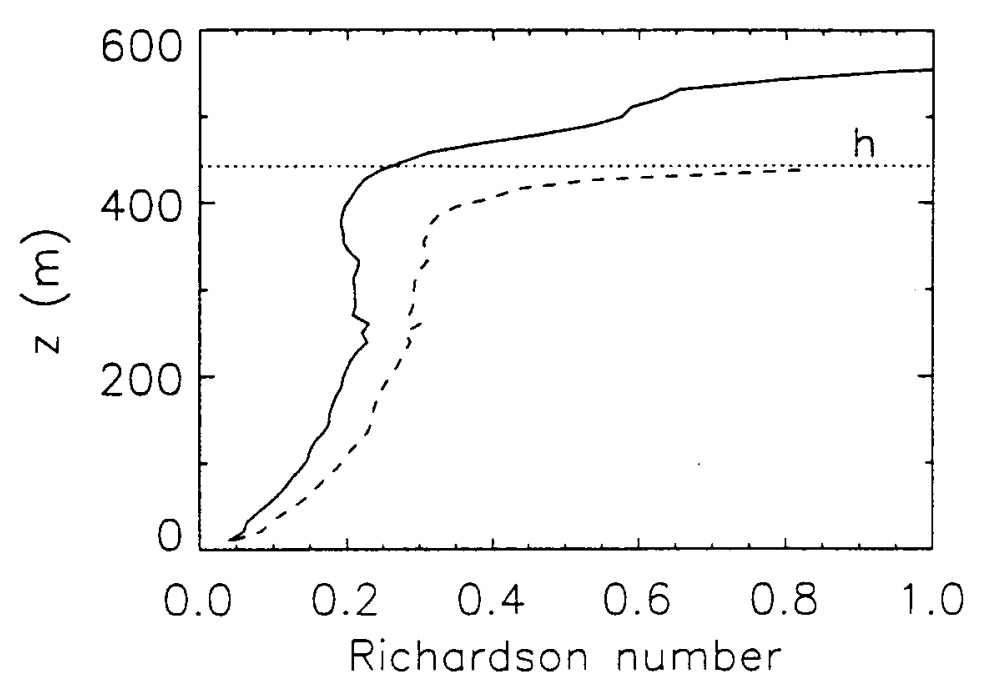

Figure 3. Vertical profiles of Richardson number. Dashed line, $\mathrm{Ri}_{f}$; solid line, $\mathrm{Ri}_{g}$.

shows that the increase in $\mathrm{Ri}_{f}$ corresponds to the more rapid decrease of the horizontal shear with height in comparison with the heat flux as shown in Figures 1 and 2. Above the boundary layer, both quantities are small such that $\mathbf{R i}_{f}$ is ill defined.

As discussed in the previous section, the original aim of the two part SGS model developed by Sullivan et al. (1994) was to improve the near surface profiles of the mean wind and temperature profiles in comparison with the $\mathrm{M}-\mathrm{O}$ similarity relations. Sullivan et al. (1994) were able to improve both wind and temperature profiles by only adding the second part to the SGS momentum flux relations (Equation (8)). The application of this model in the weakly-stable boundary-layer computations of Andren (1995) also produced improved surface-layer profiles. The modification of the model in the current study by adding a second term to the SGS heat flux (Equation (13)) did not degrade the improvement of the near surface profiles. This can be observed in Figures $4 \mathrm{a}, \mathrm{b}$ where profiles of the nondimensional gradients of velocity and temperature (Equations (11) and (14)) extracted from the LES are compared with the relations developed by Businger et al. (1971). The velocity gradient curve $\left(\phi_{m}\right)$ agrees with Businger's equation fairly well, while the temperature gradient falls below the experimental curve in a manner similar to the LES results of Brown (1994). We conclude from these plots that the modified two part version of the SGS model by Sullivan et al. (1994) continues to produce reasonable mean velocity and temperature profiles in the surface layer for the SBL.

\subsection{FLOW Visualization}

Some of the flow features of the SBL are presented in Figure 5 where instantaneous contours (after about eleven hours of cooling) of fluctuating horizontal and vertical velocity ( $u$ and $w$ ), resolved momentum flux ( $u w)$, fluctuating virtual temperature 

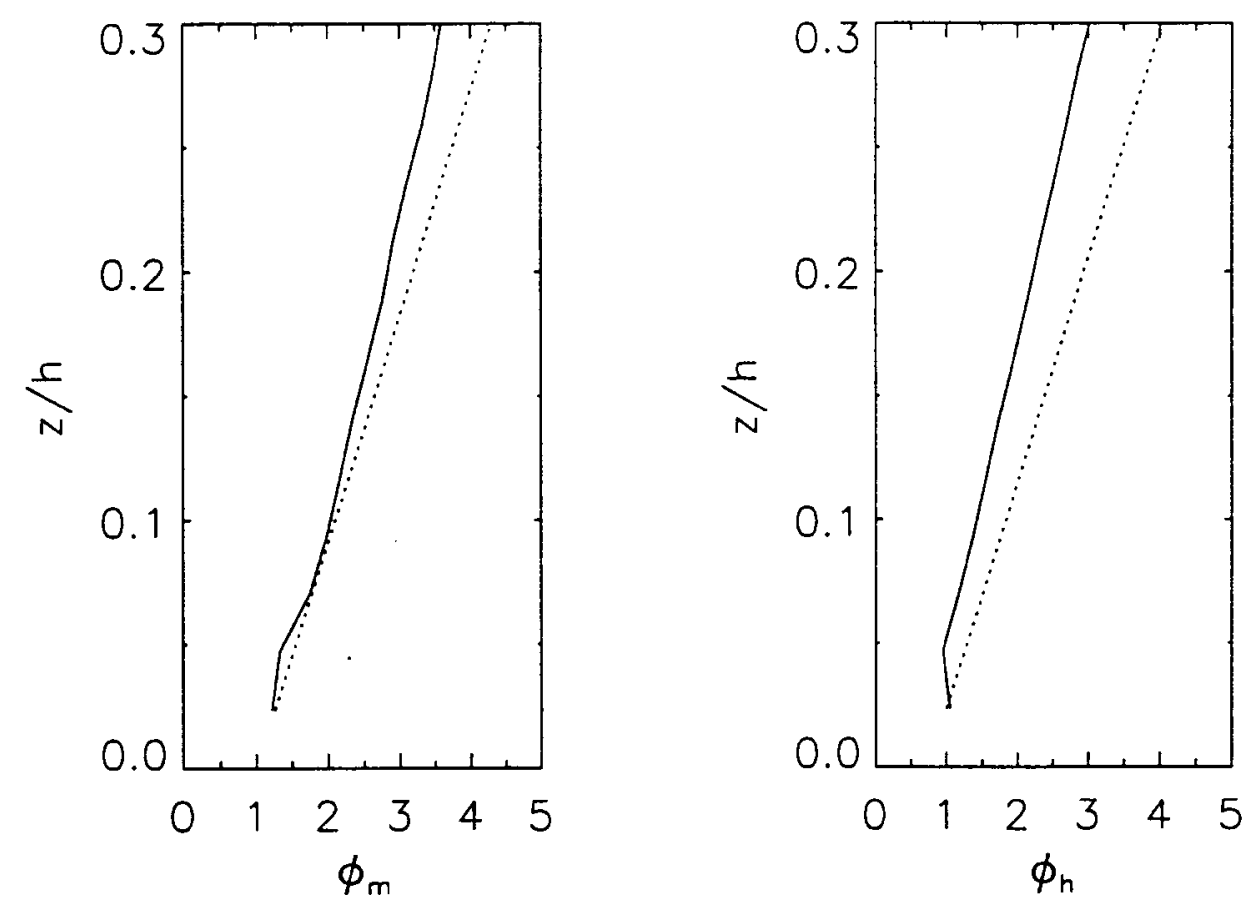

Figure 4. Profiles of nondimensional gradients. Solid line, LES results; dashed line Businger et al. (1971).

$(\theta)$, and resolved vertical heat flux in the $x-z$ plane at $y=625 \mathrm{~m}$ are plotted. These figures reveal that smaller sized structures develop in the SBL, unlike in the CBL where large plumes which span the height of the boundary layer dominate the flow field. However, an updraft/downdraft pair of small eddy structures centered around $x=400 \mathrm{~m}$ appears in Figure 5 in a manner similar to those observed in a purely shear driven boundary layer (Moeng and Sullivan, 1994). The shear boundary-layer layer calculations of Moeng and Sullivan (1994) further revealed that the eddies were roughly circular and tended to be concentrated at scales roughly one-half of the boundary-layer height, $h$. In contrast, the eddies that develop in the current stable boundary-layer simulation form at scales closer to $\frac{h}{4}$, and they exhibit a flatter shape. The ratio of the vertical to horizontal extent of the eddies in Figure 5 is $\approx 0.5$. Although the number of eddies appears patchy in Figure 5, their production is continuous. This is contrary to what we would expect for turbulence that is globally intermittent, i.e., where turbulence is suppressed over long periods of time in comparison with the time scales of the individual eddies (Mahrt, 1998). With the exception of temperature (which will be discussed further below), the amplitudes of the fluctuations are greatest for $z \leq 200 \mathrm{~m}$ where the turbulence production is strongest. 

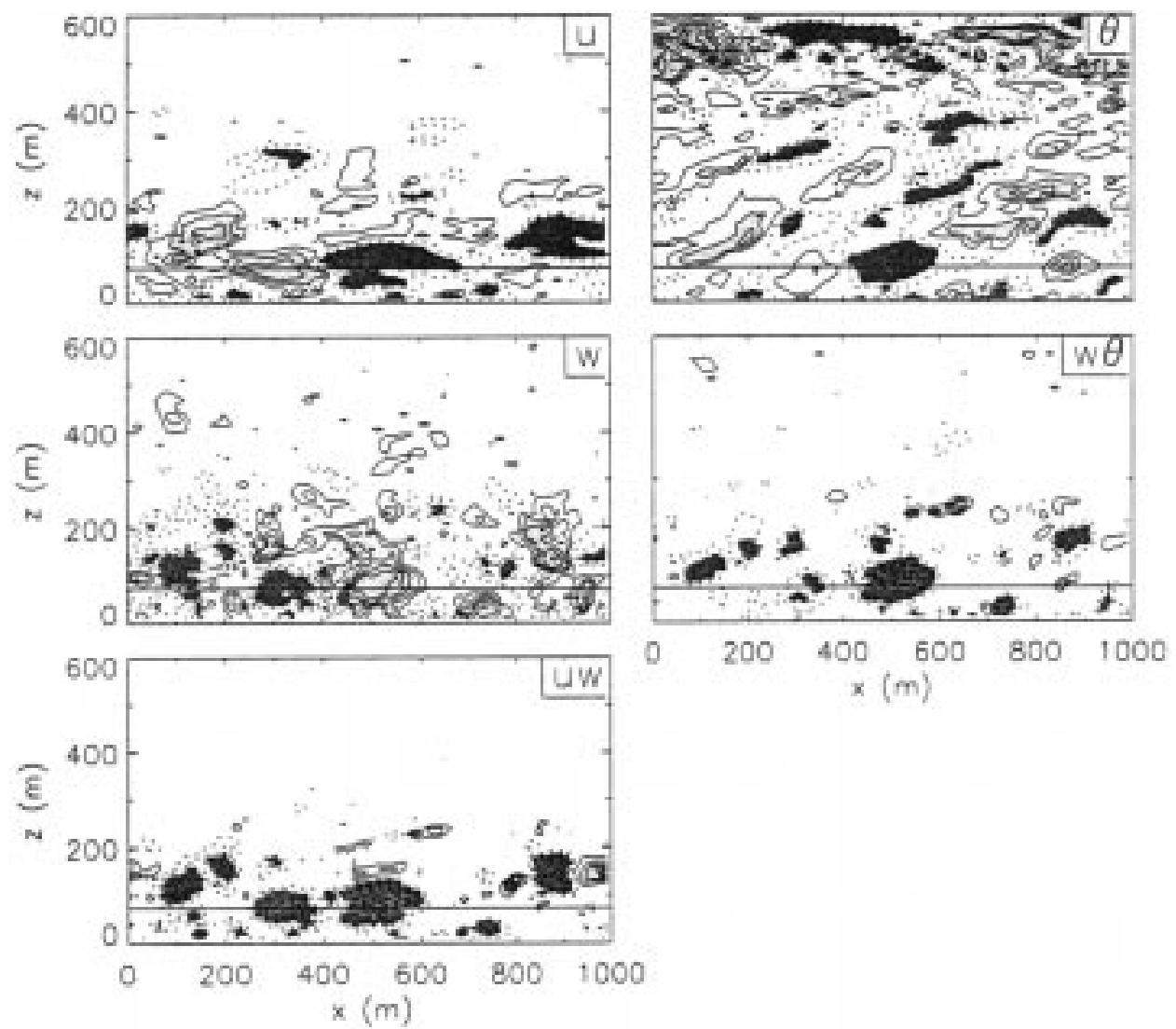

Figure 5. Contours of $u$ (range: $-2.5-1.5$, interval: 0.5 ), $w$ (range: $-1.0-1.0$, interval: 0.2), $u w$ (range: $-2.4-1.2$, interval: 0.2 ), $\theta$ (range: $-0.8-0.4$, interval: 0.1 ), and $w \theta$ (range: $-0.5-$ 0.25 , interval: 0.05 ) in the $x-z$ plane at $y=625 \mathrm{~m}$ after $\approx 11$ hours of cooling. Solid lines, positive contours; dashed lines, negative contours. Lighter and darker shaded areas highlight positive and negative extrema, respectively. The horizontal line at $z=73 \mathrm{~m}$ denotes the location of the $x-y$ plane shown in Figure 11.

The correlations in these contour plots between $w$ and $u$ that result in $u w<0$ indicate that turbulent events associated with sweeps $(w<0$ and $u>0)$ and bursts $(w>0$ and $u<0)$ are contributing equally to the turbulent fluxes. The quadrant analysis of $u w$ for the whole SBL reveals that on average the number of sweeping events is somewhat greater than the bursting events throughout the bulk of the SBL, while the strength of the bursting events is slightly greater than the sweep events (Figure 6). As a result, neither type of event dominates over the other, contrary to the neutral boundary-layer results evaluated by Lin et al. (1996) where ejections were found to prevail. The coherent structures that developed in Lin et al. (1996) consisted of horseshoe vortices that formed by one of two processes: (1) the collision between ejections and streaks, or (2) the collision of ejections with the mean flow. Flow visualization of the SBL revealed the appearance of a few 

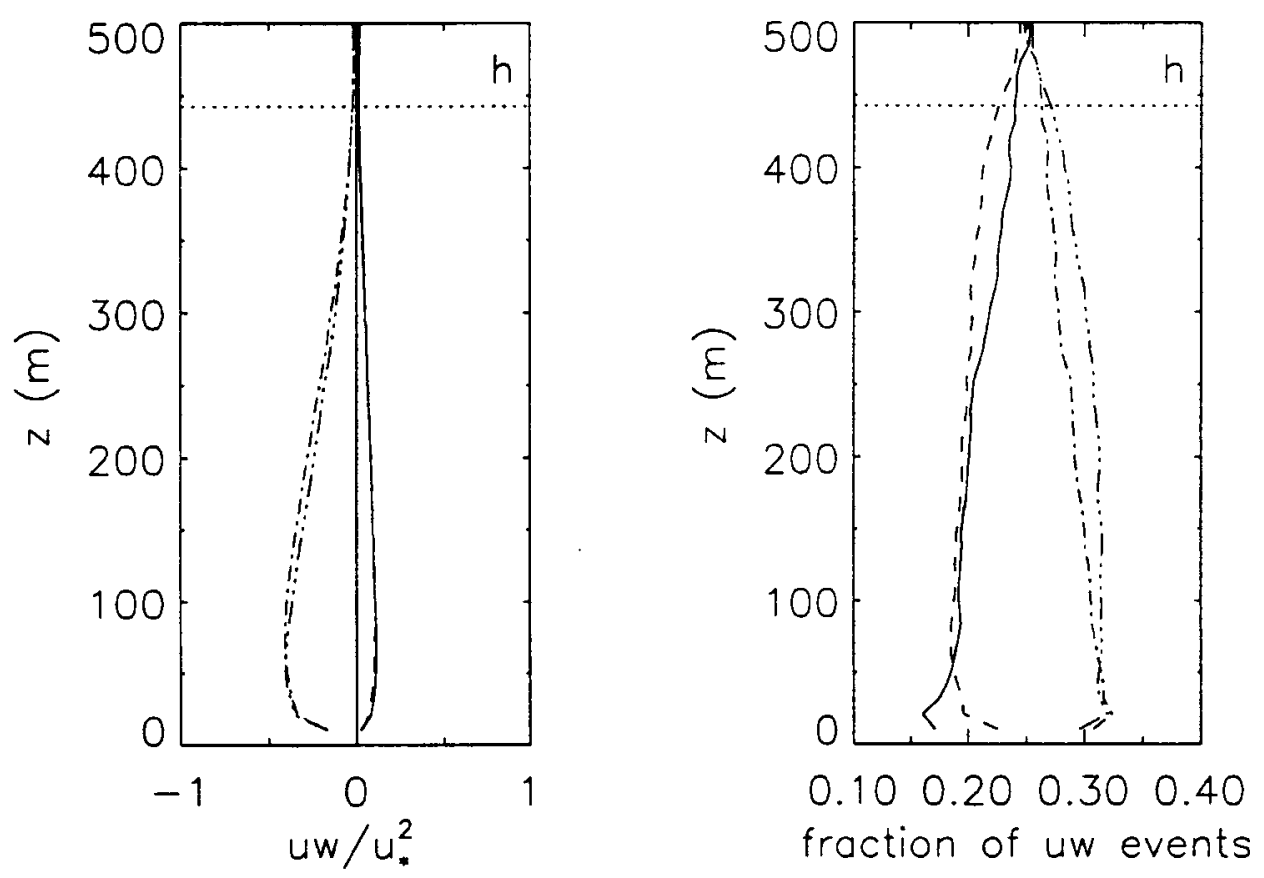

Figure 6. Profiles of $u w$ decomposed into quadrants. Solid line, $u>0$ and $w>0$; dashed-dotted line, $u<0$ and $w>0$ (ejections); dashed line, $u<0$ and $w<0$; dashed double-dotted line, $u>0$ and $w<0$ (sweeps).

coherent structures in the form of horseshoe vortices that typically formed by the second process outlined by Lin et al. (1996). Due to the cooling of the SBL, the vertical velocity is reduced throughout the boundary layer, thus events associated with ejections and sweeps are weakened and the chance of such coherent structures forming is reduced.

The quadrant analysis of $w \theta$ in Figure 7 reveals that the amplitudes of events associated with downward moving warmer air $(w<0$ and $\theta>0)$ and upward moving cooler air $(w>0$ and $\theta<0)$ are similar. Flow visualization of $w \theta$ indicates that negative heat fluxes with $w>0$ and $\theta<0$ are correlated with the development of the few horseshoe vortices observed in this case. Consequently, the formation of the horseshoe vortices can be associated with collisions of cooler air ejected from the surface with the mean flow.

As noted above, the fluctuations in potential temperature persist throughout the depth of the boundary layer reaching a maximum near $z=500 \mathrm{~m}$ where the strong capping inversion appears (Figure 5). Focusing on this region, we observe that the temperature fluctuations correspond to the presence of a gravity wave, which is denoted by an upstream cell of $\theta<0$ and a downstream cell of $\theta>0$. This wave can be seen in a clearer manner in both temperature and vertical velocity fluctuations by averaging these quantities across the computational domain at constant $x$ 

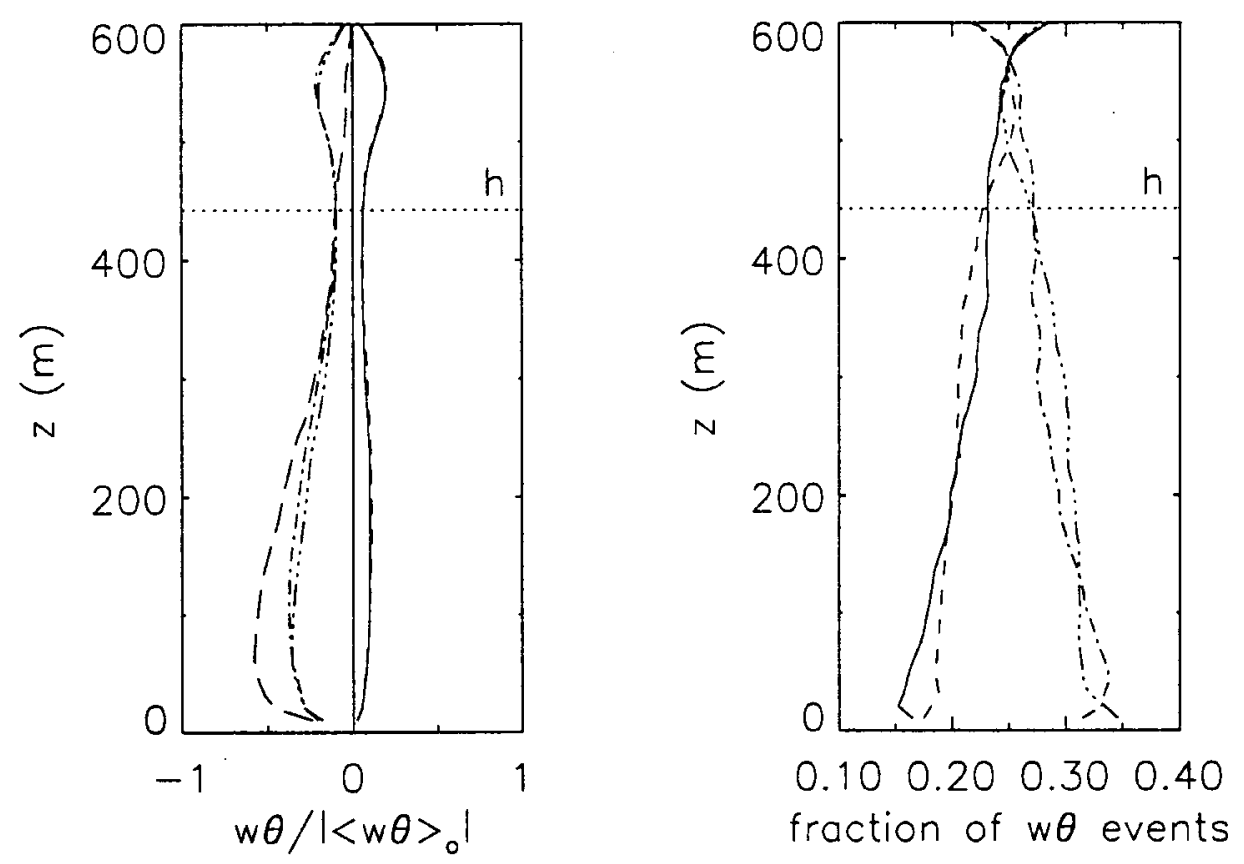

Figure 7. Profiles of $w \theta$ decomposed into quadrants. Solid line, $w>0$ and $\theta>0$; dashed-dotted line, $w>0$ and $\theta<0$ (rising cool air); dashed line, $w<0$ and $\theta<0$; dashed double-dotted line, $w<0$ and $\theta>0$ (descending warm air); long dashed line, total $w \theta$.
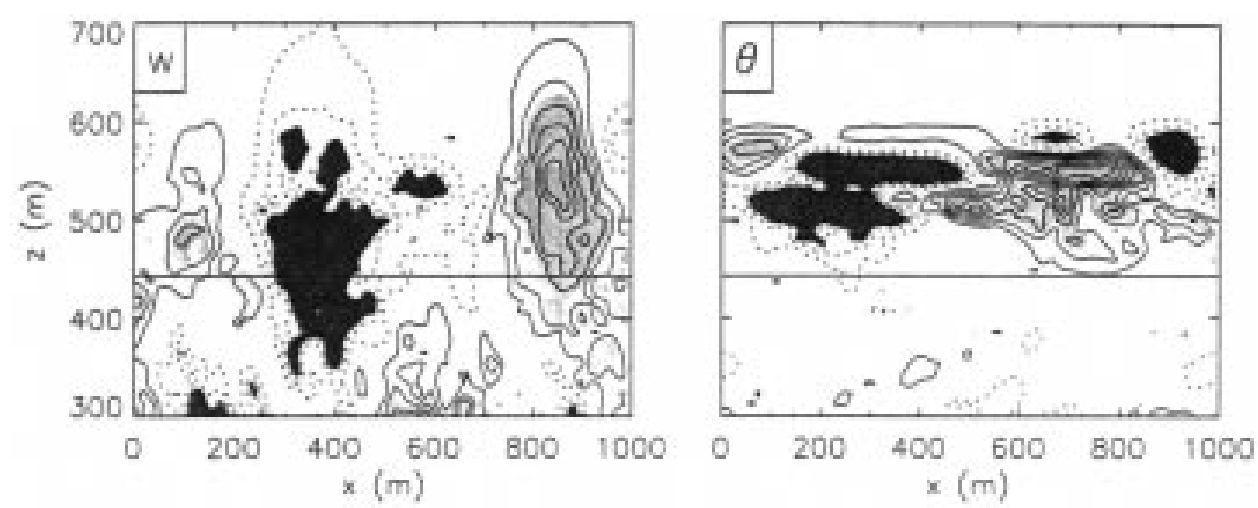

Figure 8. Contours of conditionally averaged $w$ (range: $-0.12-0.12$, interval: 0.02$)$ and $\theta(-0.28$ -0.32 , interval: 0.04 ) in the $x-z$ plane after $\approx 11$ hours of cooling. Solid lines, positive contours; dashed lines, negative contours. Lighter and darker shaded areas highlight positive and negative extrema, respectively. The horizontal line denotes the height of the boundary layer, $h$. The $z$ direction is magnified two times its normal size. 


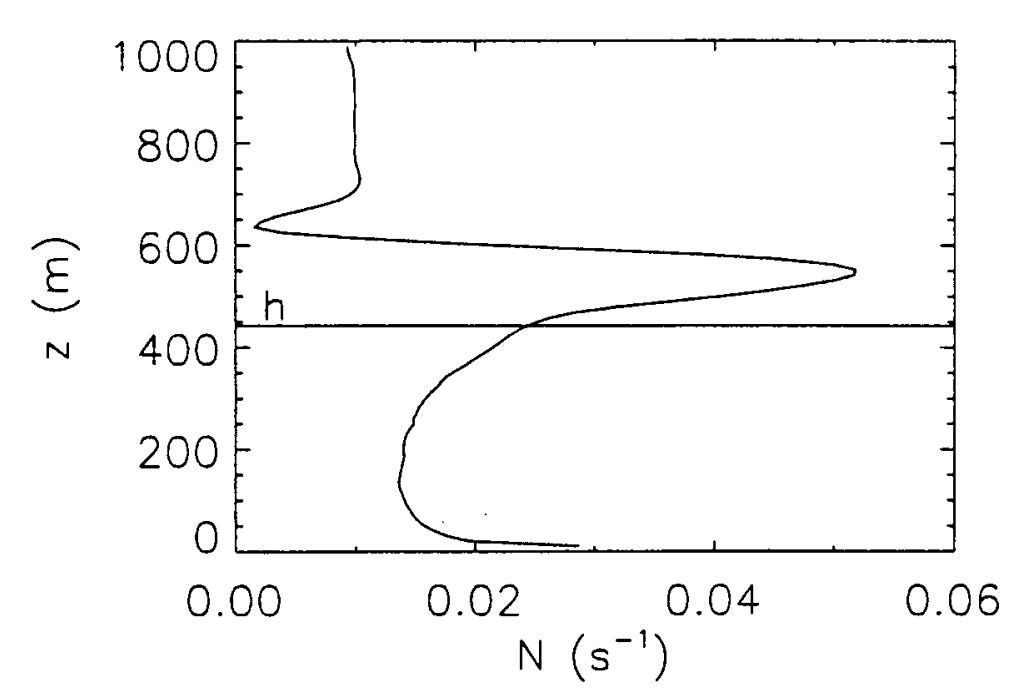

Figure 9. Vertical profile of the Brunt-Väisälä frequency.

(Figure 8 ). The $w$ contours corresponding to the wave near $z=500 \mathrm{~m}$ are roughly $90^{\circ}$ out of phase with the temperature contours. If $w$ and $\theta$ were exactly $90^{\circ}$ out of phase, the heat flux and the correlation coefficient between $w$ and $\theta$ would be zero. However, the correlation coefficient in the region spanning $z=500$ and $600 \mathrm{~m}$ is $\approx 0.1$ and Figure 1 reveals that $w \theta$ is small but nonzero above the top of the boundary layer indicating that the wave behaviour is weakly nonlinear.

The contours in Figure 8 further show that the wave develops primarily between the top of the boundary layer $(z=442.5 \mathrm{~m})$ and the top of the capping inversion region $(z=600 \mathrm{~m})$. The vertical distribution of the Brunt-Väisälä frequency $(N)$ presented in Figure 9, indicates that any waves that propagate vertically upward from below have frequencies smaller than $N_{\max }=0.052 \mathrm{~s}^{-1}$ at $z=550 \mathrm{~m}$ (we suspect that the minimum in $N$ near $z \approx 640 \mathrm{~m}$ is a numerical artifact associated with our implementation of the monotone discretization scheme). Above $z=550 \mathrm{~m}$, waves with higher frequencies will be evanescent as they exceed the Brunt-Väisälä frequency of the upper layer.

Within the boundary layer, the turbulence is sufficiently strong enough to prevent any waves from being easily discerned. The spectra in the boundary layer are smooth and there is no evidence of distinctive peaks at low frequencies to indicate the presence of waves. A peak corresponding to the wave above the boundary layer does appear in the spectra for vertical velocity around $z=350 \mathrm{~m}$ in accordance with Figure 8 , where the $w$ contours associated with the gravity wave extend below the height of the SBL. The penetration of the wave into the boundary layer reflects the relative weakness of the turbulence at the top.

Based upon a rough tracking of the time needed for the troughs and crests of the wave to traverse the computational domain, we estimate the phase speed of 
the wave to be $c \approx 6.1 \mathrm{~m} \mathrm{~s}^{-1}$, and, by assuming a frequency of $N=0.052 \mathrm{~s}^{-1}$, the wavelength can be determined as $\lambda=\frac{2 \pi c}{N}=737 \mathrm{~m}$. An estimation of the wavelength directly from Figure 8 is somewhat larger at $\lambda \approx 800 \mathrm{~m}$. The fact that these estimated wavelengths are fairly near the size of the computational domain $\left(L_{x}=1000 \mathrm{~m}\right)$, begs the question as to whether the preferred wavelength is actually longer and the size of the computational domain is artificially forcing the wavelength. The estimated phase speed of the wave is not equal to the local mean speed of the flow indicating that even though shear is present due to the low-level jet, the wave is not a Kelvin-Helmholtz $(\mathrm{K}-\mathrm{H})$ wave. In addition, the gradient Richardson number in the region where the wave develops is greater than the critical value for $\mathrm{K}-\mathrm{H}$ instability (Figure 3 ). A K-H wavelength estimated with $c \approx U_{g}$ and frequency $\omega=0.052 \mathrm{~s}^{-1}$ would be several kilometres, which would clearly exceed the size of the domain.

To determine if the wavelength is a physical manifestation of the flow field, we consider the linear stability equation for gravity waves, the Taylor-Goldstein equation (Gossard and Hooke, 1975),

$$
W^{\prime \prime}(z)+\left(\frac{N(z)^{2}}{(U(z)-C)^{2}}-\frac{U^{\prime \prime}(z)}{(U(z)-C)}-\alpha^{2}\right) W(z)=0 .
$$

Here, $W$ is the vertical fluctuating velocity component in Fourier space, $C$ is the complex phase speed $\left(C=c+i c_{i}\right)$ of a wave with wavenumber, $\alpha=\frac{2 \pi}{\lambda}$, and the symbol ' denotes the derivative with respect to $z$. It should be mentioned that this equation describes the linear behaviour of small amplitude waves. The wave observed in the current study exhibits nonlinear characteristics, however as noted above they appear to be weak, and therefore we feel this analysis is valid for our purposes.

Given the wavenumber and horizontally averaged profiles for $N$ (Figure 9) and $U$ (Figure 10), $C$ can be determined as a complex eigenvalue of Equation (23) (see Appendix A for the numerical algorithm used to solve Equation (23)). The real and imaginary parts of $C$ define the phase speed, $c$, and growth rate, $c_{i}$, of the wave. This is an inviscid problem, therefore all waves are either unstable $\left(c_{i}>0\right)$ or neutrally stable $\left(c_{i}=0\right)$ (Hazel, 1972; Gossard and Hooke, 1975). The frequency of the wave, $\omega$, can be determined from the real part of $C$ as $\omega=\frac{2 \pi c}{\lambda}$.

The outcome of using this equation to evaluate the wave that develops above the SBL is summarized in Table II, where we present the eigenvalue with the highest growth rate obtained for three different wavelengths: the estimated wavelengths, $\lambda=737$ and $800 \mathrm{~m}$, and a wavelength equal to the size of the computational domain, $\lambda=1000 \mathrm{~m}$. Note that the wave speeds, $c$, determined from this analysis for each wavelength $\left(c=6.38,6.37\right.$, and $\left.6.36 \mathrm{~m} \mathrm{~s}^{-1}\right)$, are close to the wave speed roughly estimated above $\left(c \approx 6.1 \mathrm{~m} \mathrm{~s}^{-1}\right)$. In addition, the resulting frequencies for wavelengths $\lambda=737$ and $800 \mathrm{~m}$ lie close to the maximum value of $N=0.052 \mathrm{~s}^{-1}$ at $z=550 \mathrm{~m}$, whereas the frequency for $\lambda=1000 \mathrm{~m}$ is much smaller. 


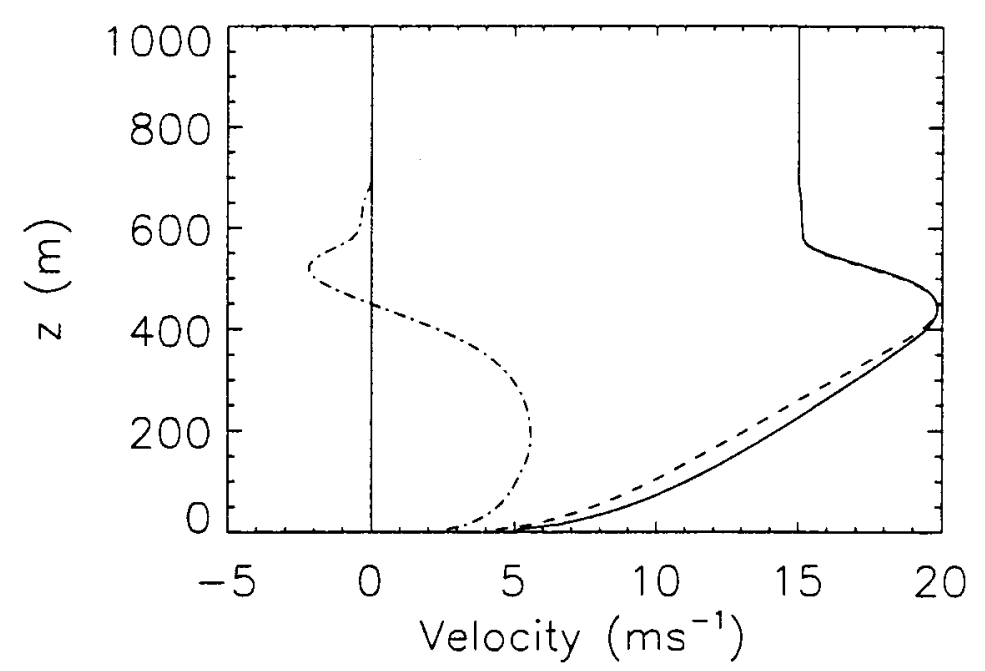

Figure 10. Vertical profiles of velocity. Dashed line, U; dashed-dotted line, V; solid line, $M=\left(U^{2}+V^{2}\right)^{\frac{1}{2}}$.

\section{TABLE II}

Results of solving Equation (23) for different wavelengths using the Brunt-Väisälä profile in Figure 9 and mean velocity profile in Figure 10.

\begin{tabular}{rll}
\hline$\lambda(\mathrm{m})$ & $C\left(\mathrm{~m} \mathrm{~s}^{-1}\right)$ & $\omega\left(\mathrm{m} \mathrm{s}^{-1}\right)$ \\
\hline 737 & $6.38+i 0.267$ & 0.055 \\
800 & $6.37+i 0.273$ & 0.05 \\
1000 & $6.36+i 0.29$ & 0.04 \\
\hline
\end{tabular}

These results show that the wavelength of the gravity wave observed in the current computation has not been truncated by the size of the computational domain. This analysis also reveals that the dominant gravity wave that develops above the stable boundary layer is linked to the most unstable wave, i.e., the wave with the highest growth rate, $c_{i}$, as predicted by the Taylor-Goldstein wave equation. It appears that this wave originates in the SBL where it propagates vertically until it reaches the capping inversion. The wave is then trapped at the inversion as it cannot propagate further into the weaker inversion above. The same analysis performed on results from additional SBL LESs, where the strength of cooling and the geostrophic wind were varied, revealed the same conclusion. We observed that the wavelength typically decreased with decreasing geostrophic wind, whereas there was no obvious trend in the wavelength for different cooling fluxes. In each 
of the cases we examined, the capping inversion was quite strong. With a weaker capping inversion (smaller $N$ ) the most unstable wave would not be trapped, but would continue to propagate vertically as observed in the case of Andren (1995).

This link between the waves in the inversion and the Taylor-Goldstein equation was not observed for either the convective or neutral boundary layer. In these cases, waves cannot develop within the boundary layer, but can form above if a stably stratified capping inversion is present. However, unlike the SBL, the turbulence in the CBL and neutral boundary layer persists all the way up to the capping inversion, and will influence the development of the waves. This behaviour has been considered theoretically by Carruthers and Moeng (1987) for the CBL where the turbulent velocity and length scales have been used to predict the wavelength and frequency of the resonant waves in the inversion. In the current study, the gravity wave itself does not appear to have a marked effect on the turbulence. This is in contrast with the observations of Finnigan et al. (1984) where it was observed that turbulence was maintained by energy transfer from gravity waves in the SBL.

To conclude this section, we briefly present contours of $u, w, \theta, u w$ and $w \theta$ in the $x-y$ plane near the surface at $z=73 \mathrm{~m}$ (Figure 11). In the neutral boundary layer, the near surface region is typically characterized by the appearance of alternating streaks of high and low speed fluid aligned in the mean flow direction, while in the mixed convective/neutral boundary layer, the streaks coexist with horizontal rolls. Figure 11 shows that there are a few small streaky-like structures that develop in the SBL. The $u$ contours reveal a pair of high $(u>0)$ and low $(u<0)$ speed streaks that are nearly aligned in the mean flow direction near $y=600 \mathrm{~m}$ and which correspond to the weak updraft/downdraft pair of eddies observed in the crossplane shown in Figure 5. The $w$ fluctuations reflect stronger streaky behaviour associated with the low speed $u$ streak resulting in the development of one $u w$ streak near $y=600 \mathrm{~m}$. Accordingly, streak-like regions where $\theta<0$ and $w \theta<0$ also form only at the same location as the low speed $u$ streak. These contours further emphasize the reduction in the size of the structures that develop in the SBL due to the cooling.

\subsection{LOW-LEVEL JET}

The most interesting feature of the present simulation is the formation of a lowlevel jet at the top of the boundary layer that develops due to the inertial oscillation associated with the Coriolis force. The jet can be clearly observed in Figure 10 where the mean velocity profile exhibits a supergeostrophic maximum of $M=$ $\left(U^{2}+V^{2}\right)^{\frac{1}{2}} \approx 20.3 \mathrm{~m} \mathrm{~s}^{-1}$ at the top of the SBL near $z \approx 450 \mathrm{~m}$. The dependency of the jet on the Coriolis force can be observed by considering the horizontal momentum equations for the mean wind components, $U$ and $V$, 


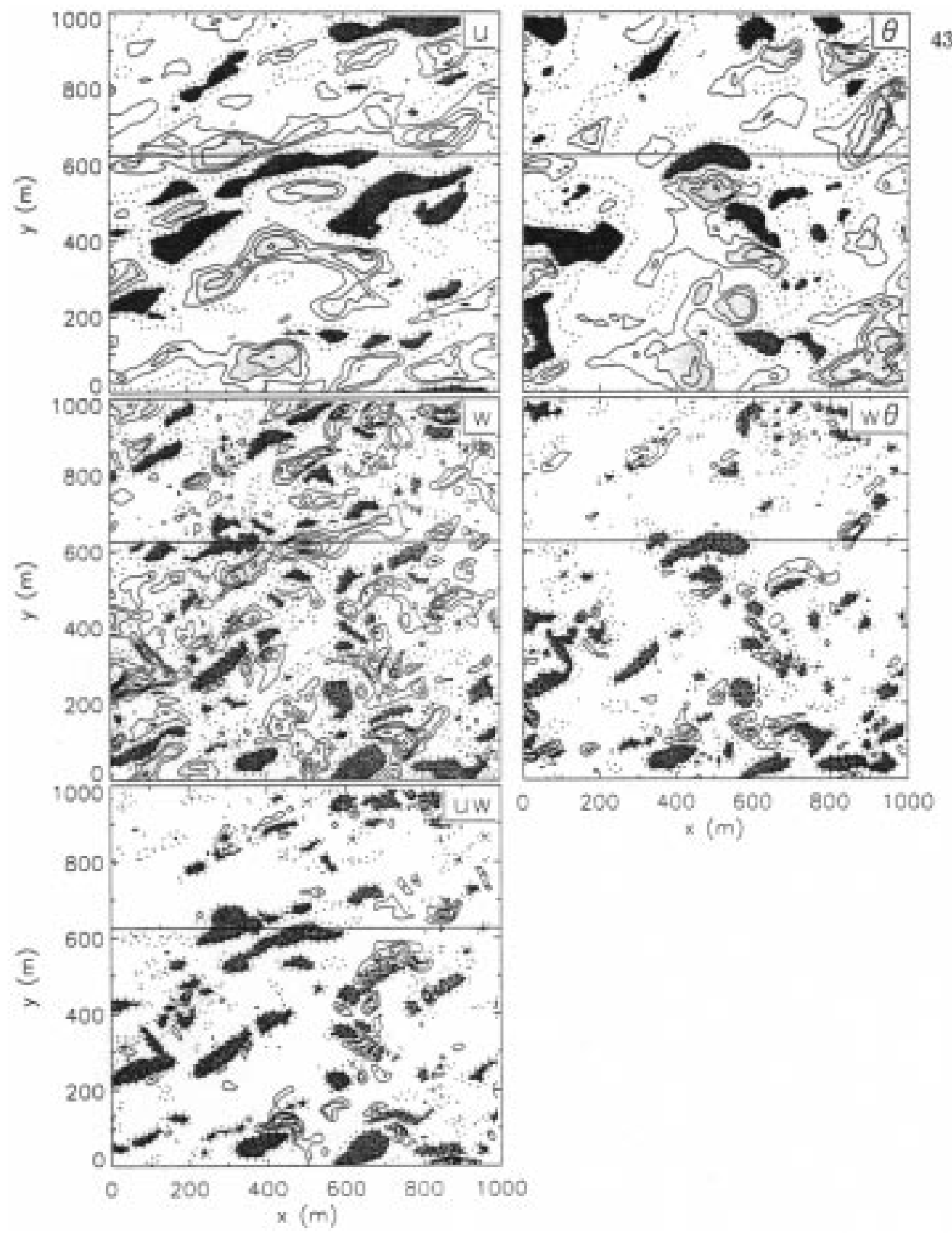

Figure 11. Contours of $u$ (range: $-3.0-2.5$, interval: 0.5 ), $w$ (range: $-2-1.75$, interval: 0.25 ), $u w$ (range: $-3.5-1.75$, interval: 0.25 ), $\theta$ (range: $-0.4-0.6$, interval: 0.1 ), and $w \theta$ (range: -0.65 -0.2 , interval: 0.05$)$ in the $x-y$ plane at $z=73 \mathrm{~m}\left(\frac{z}{h}=0.16\right)$ after $\approx 11$ hours of cooling. Solid lines, positive contours; dashed lines, negative contours. Lighter and darker shaded areas highlight positive and negative extrema, respectively. The horizontal line at $x=625 \mathrm{~m}$ denotes the location of the cross-plane shown in Figure 5. 


$$
\begin{aligned}
& \frac{\partial U}{\partial t}=-\frac{\partial\langle u w\rangle}{\partial z}+f V \\
& \frac{\partial V}{\partial t}=-\frac{\partial\langle v w\rangle}{\partial z}-f\left(U-U_{g}\right) .
\end{aligned}
$$

Here, $f=1 \times 10^{-4} \mathrm{~s}^{-1}$, is the Coriolis parameter at a latitude of $\phi=45^{\circ}$. As the boundary layer cools, the turbulence at the top collapses such that $\langle u w\rangle$ and $\langle v w\rangle$ decrease. To compensate for this collapse and to balance the Coriolis terms of Equation (24), $U$ must increase beyond $U_{g}$.

The behaviour of the winds associated with the nocturnal jet was first examined closely by Blackadar (1957). His analysis demonstrated that if the turbulent stresses fall to zero, the velocity of the jet will oscillate around the geostrophic velocity, $U_{g}$, with a frequency of $f$. This can be seen by recasting the difference between the mean horizontal velocity components and the geostrophic velocity as a complex number, i.e., $W=\left(U-U_{g}\right)-i V$, setting the stress terms to zero, and then rewriting Equation (24) as

$$
\frac{\partial W}{\partial t}=-i f W .
$$

The solution to the above equation is simply,

$$
W=W_{o} e^{-i f t},
$$

where $W_{o}$ is the ageostrophic velocity at the time when the cooling of the boundary layer begins. This idealized equation reflects the undamped oscillatory behaviour of the jet, as well as the fact that the maximum amplitude of the jet is dependent upon the initial deviation of the wind field from the geostrophic velocity. The hodograph formed by the velocity components obtained from Equation (26) is a circle with radius, $\left|W_{o}\right|=\left(\left(U-U_{g}\right)^{2}+V^{2}\right)^{\frac{1}{2}}$.

The time evolution of the maximum velocity of the jet $\left(U_{j}\right)$ that develops in the current simulation is contrasted with the prediction of $U_{j}$ given by Equation (26) in Figure 12. In these plots, time is measured from the moment when the cooling begins with the application of the smallest negative flux $\left(\langle w \theta\rangle_{o}=-0.02 \mathrm{~m} \mathrm{Ks}^{-1}\right)$ to the boundary layer. The LES results for $U_{j}$ were obtained by tracking the maxima in the profiles of $M$ in time, thus in the early stages of cooling this value was simply equal to $U_{g}$ up until the point in the simulation where the effects of the cooling were felt aloft and $M_{\max }=U_{j}$ became supergeostrophic. The time evolution of the jet velocity predicted by Equation (26) is computed using $W_{o}=(-4,3.55) \mathrm{m} \mathrm{s}^{-1}$, which is the geostrophic departure of the mean velocities measured at the final height of the jet, $z=450 \mathrm{~m}$, at the time when the cooling begins. Figure 12 reveals that $M_{\max }=U_{j}$ in the LES exceeds $U_{g}$ after about two hours of cooling. This is then followed by a steady increase over about seven hours reaching a maximum 


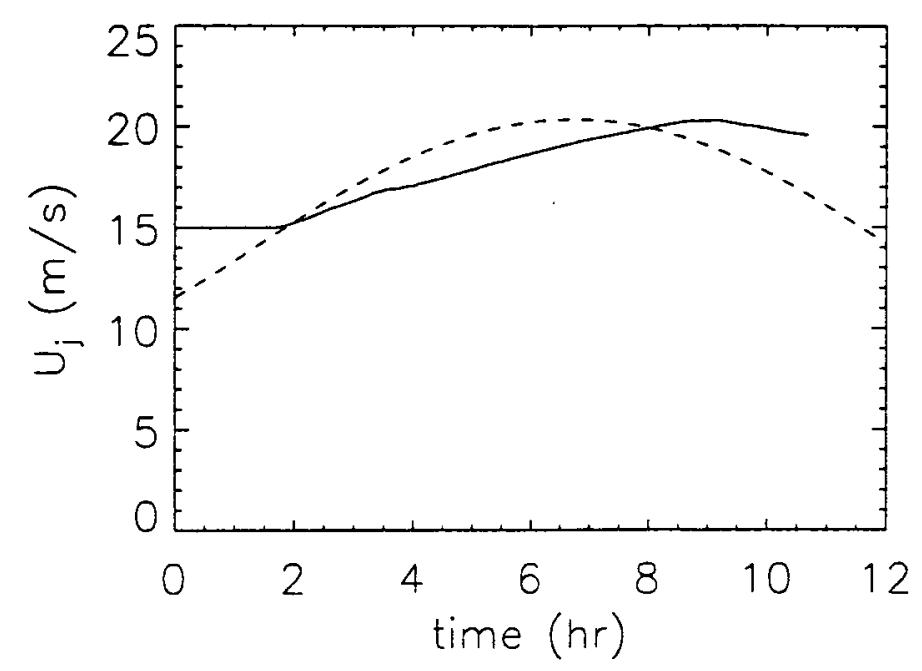

Figure 12. Time evolution of the maximum low-level jet velocity. Time is measured from when the cooling first begins. Solid line, LES results; dashed line, solution from Equation (26) with $W_{o}=(-4$, $3.55) \mathrm{m} \mathrm{s}^{-1}$.

value of $U_{j} \approx 20.3 \mathrm{~m} \mathrm{~s}^{-1}$ after nine hours of cooling. Beyond this point, $U_{j}$ decreases in accordance with the effects of the inertial oscillation. The prediction given by the dashed curve in Figure 12 reflects the idealization of the boundary layer used to obtain Equation (26). In particular, it assumes that the turbulent stresses fall to zero instantaneously, and as a result the peak in the maximum value of $U_{j}$ develops earlier. As noted above, the LES results show that there is an initial period of two hours during which the effects of the cooling permeate the boundary layer followed by the decay of the turbulent stresses at the top. A shift of the dashed curve predicted by Equation (26) by two hours results in an improved comparison of the occurrence of the maximum $U_{j}$ with the LES curve in Figure 12.

The time evolution of the height of the jet is presented in Figure 13. When $U_{j}$ becomes geostrophic after two hours of cooling, the height of the maximum velocity shifts downward to just below the level of the capping inversion and then slowly decreases from $500 \mathrm{~m}$ to $450 \mathrm{~m}$. The gradual cooling of the SBL in our LES required increases in the magnitude of the downward surface heat flux at $t \approx 4,5$, and $6 \mathrm{hr}$, however there does not appear to be a marked effect on the time development of either $h_{j}$ or $U_{j}$ due to these increases. The time-evolution behaviour of the height and velocity of the jet is qualitatively similar to the one-dimensional model results of Delage (1974).

Figure 14 presents the hodograph of the ageostrophic velocity components of the jet obtained from the LES. For clarity in the following discussion, sections of the hodograph have been broken down into three parts denoted as I, II, and III and the location of the maximum jet velocity is highlighted by a $\diamond$. These results are further compared with the predicted jet velocity components given by 


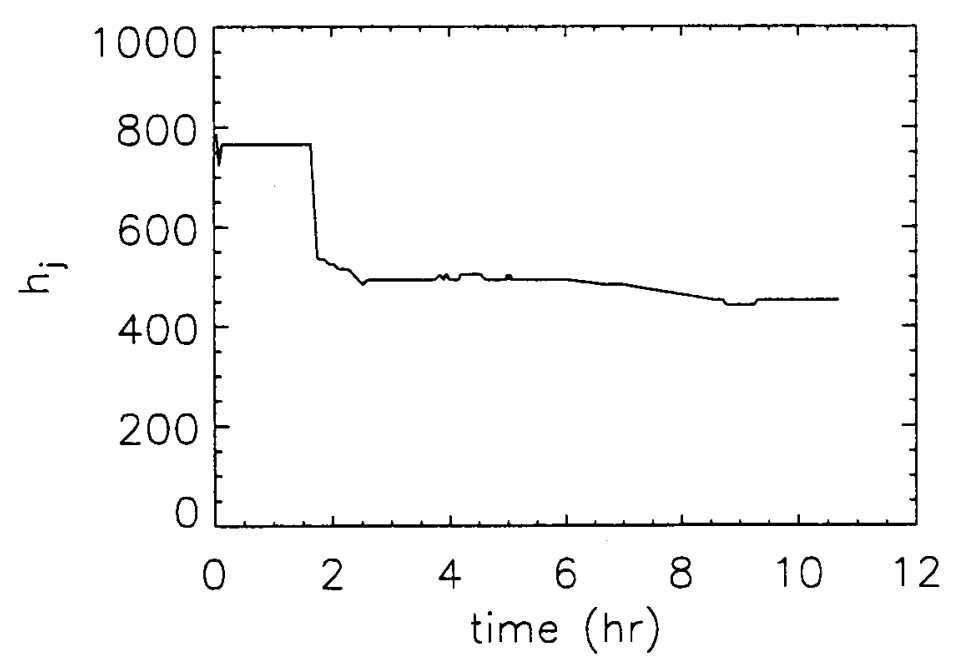

Figure 13. Time evolution of the height of the low-level jet.

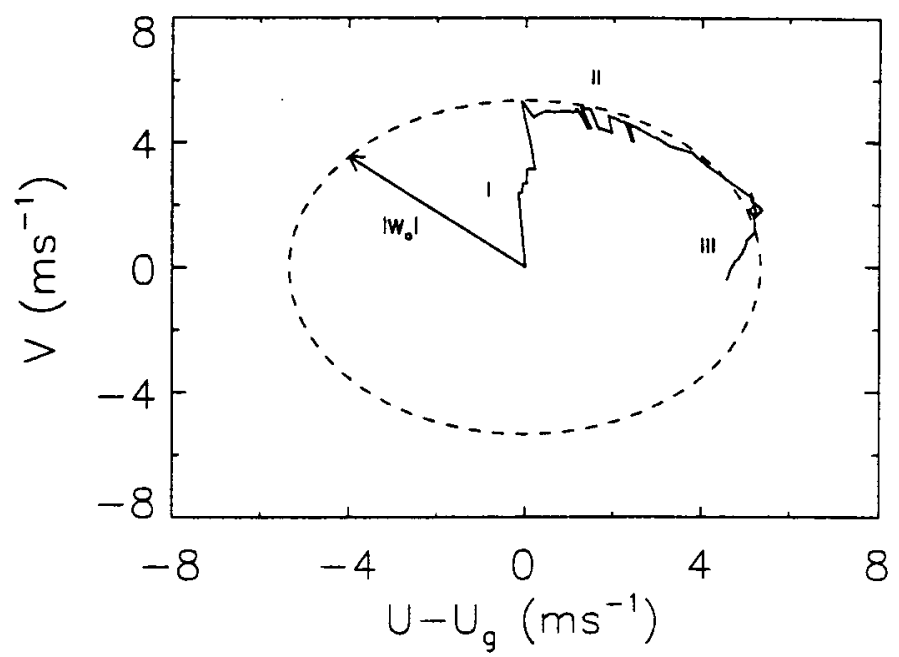

Figure 14. Hodographs of the low-level jet ageostrophic velocity components. Solid line, LES result; dashed line, solution from Equation (26) with $W_{o}=(-4,3.55) \mathrm{m} \mathrm{s}^{-1}$. The $\diamond$ denotes the point where the maximum velocity of the jet develops in the LES.

Equation (26). The first portion of the LES curve (section I) reflects the two hour pre-development stage when no jet is present. As the turbulence aloft decays due to the cooling, the jet begins to develop and, in accordance with the influence of the inertial oscillations, the hodograph forms a quarter circle in the upper right hand quadrant of Figure 14 (sections II and III). The hodograph of the LES results in section II compares quite well with Equation (26) up to the point where the jet maximum occurs. Although the predicted value of the maximum jet speed 


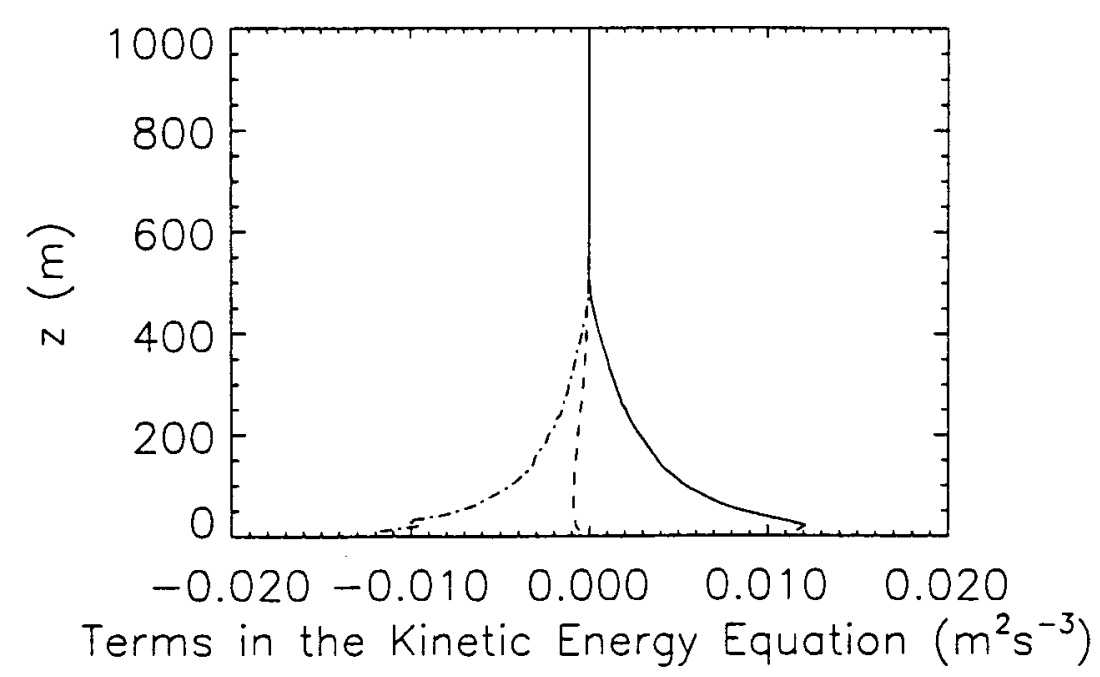

Figure 15. Vertical profiles of the dominant turbulent kinetic energy budget terms. Solid line, shear production; dashed line, buoyancy; dashed-dotted line, dissipation.

$\left(20.35 \mathrm{~m} \mathrm{~s}^{-1}\right)$ agrees extremely well with the observed LES value $\left(20.3 \mathrm{~m} \mathrm{~s}^{-1}\right)$, the locations of the maxima on the hodographs are different. The idealized behaviour described by Equation (26) requires that the maximum in the jet velocity occurs when $V=0 \mathrm{~m} \mathrm{~s}^{-1}$, however this is clearly not the case for the LES results as the jet maximum occurs when the ageostrophic velocity components of the jet are $\left(U-U_{g}, V\right)=(5.22,1.82) \mathrm{m} \mathrm{s}^{-1}$ (as denoted by the $\diamond$ in Figure 14).

The above study comparing the development of the low-level jet observed in the LES with the simple analysis of Blackadar (1957) for describing the initial jet behaviour shows some quantitative agreement. This is possibly due to the relatively high cooling flux applied in this case which causes the turbulent stresses at the top of the boundary layer to fall close to zero fairly rapidly (after two hours of cooling). However, it should be emphasized that Equation (26) reflects an idealized situation which qualitatively describes the jet behaviour based upon the initial geostrophic departure of the wind and the assumption that the stresses are zero. We found that the behaviour of the jet did not correlate as well quantitatively with Equation (26) in other SBL cases where the cooling flux was weaker and the decay rate of the turbulent stresses was smaller.

The low-level jet may be important in the SBL as shear associated with the jet is a possible source of turbulence production aloft (Mahrt, 1998). The question of whether the low-level jet in our LES flow is producing significant turbulence can be addressed by considering the behaviour of the turbulent kinetic energy budget (Figure 15). The vertical profile of the production term in Figure 15 does not reveal a substantial effect of the jet as it decreases monotonically to zero upward from the surface. While the underside of the jet itself has considerable shear $\left(\frac{\partial U}{\partial z}\right)$, the 
amplitudes of $\langle u w\rangle$ and $\langle v w\rangle$ near the bottom of the jet are smaller than those closer to the surface (Figure 2). This decrease of $\langle u w\rangle$ with height is also reflected in the contours of Figure 5. In addition, the Richardson number profiles (Figure 3 ) do not indicate production of turbulence as $\mathrm{Ri}_{g}$ exceeds $\left(\mathrm{Ri}_{g}\right)_{c}=0.25$ at the height of the jet. Therefore, it appears that the shear associated with the jet is not a source of turbulent production in this simulated SBL. Such a jet would have a greater effect on a highly intermittent stable boundary layer where the jet induced turbulence aloft would be detached from the surface (Mahrt, 1998). The strong inversion above the SBL is also 'capping' the turbulence production aiding in the prevention of turbulence production due to the jet.

\section{Discussion and Conclusions}

In this paper, results from a large-eddy simulation of a stably stratified boundary layer were presented. The early stages of this study revealed that the two part SGS model of Sullivan et al. (1994) in its original form produced unphysical profiles in the turbulent quantities when the boundary layer was cooled by a large negative heat flux. This behaviour first manifested itself when the SGS vertical heat flux fell to zero near the surface in direct conflict with assumptions associated with largeeddy simulation of the atmosphere. This was remedied by adding a second term to the SGS vertical heat flux model in a manner similar to the model for the SGS momentum flux. While this improved SGS model alleviated these problems, the simulations were still sensitive to rapid cooling by the sudden application of large negative heat fluxes at the surface. Therefore, the boundary layer was gradually cooled by incrementally increasing the surface cooling flux.

The case investigated here involves a SBL with a geostrophic wind of $U_{g}=$ $15 \mathrm{~m} \mathrm{~s}^{-1}$, which is cooled by a maximum heat flux of $\langle w \theta\rangle_{o}=-0.05 \mathrm{~m} \mathrm{~K} \mathrm{~s}^{-1}$. The boundary layer was capped by a strong inversion at $z=500 \mathrm{~m}$. Typical turbulent profiles were presented revealing the development of a weakly stable boundary layer where the turbulence remains fairly continuous. The distributions of nondimensional gradients of velocity and temperature compared with MoninObukhov theory demonstrated that the modification of the SGS model was able to produce reasonable surface-layer profiles.

The small-scale features of the SBL were observed through flow visualization of various quantities. Quadrant analysis of the momentum and heat fluxes showed that the occurrence of structures associated with cool air ejections is similar to that of warm air sweeps. A distinctive gravity wave develops at the top of the SBL in the strong capping inversion region that penetrated into the top part of the boundary layer. Our results showed that the gravity wave is related to the wave with the highest growth rate as predicted by the Taylor-Goldstein equation. The wave develops in the SBL due to the stable stratification and then propagates upward where it is trapped by the capping inversion. A strong low-level jet of $U_{j}=$ 
$20.3 \mathrm{~m} \mathrm{~s}^{-1}$ also formed at the top of the boundary layer in conjunction with the decay of turbulent stresses and the inertial oscillation associated with the Coriolis forces. The jet velocity grew in time to a maximum value of $U_{j}=20.3 \mathrm{~m} \mathrm{~s}^{-1}$ and then began to decay while the height of the jet decreased to a level of $z=450 \mathrm{~m}$. The oscillatory behaviour of the jet showed qualitative and some quantitative agreement with the analysis of Blackadar (1957). The jet itself did not generate substantial turbulence aloft in the simulated boundary layer.

This work involves a first step towards examining a very stable boundary layer as defined by Mahrt (1998), where turbulence is highly intermittent and horizontal layering can develop. In pushing the limits of a well established SGS model that has been implemented in a number of LES investigating the atmospheric boundary layer, weaknesses of the model were exposed. We were able to avoid these problems, but ultimately to simulate the very stable boundary layer, a better SGS model that is designed for the SBL is needed. This would include gaining a better understanding of the dependence of the SGS length scale, $l$, on local stability and/or improving LES in the near surface region. The current formulation of $l$ was 'borrowed' from the work of Deardorff (1980) who developed the stability corrected relation for $l$ (Equation (7)) for simulating the capping inversion above the boundary layer and not for the stable boundary layer in particular. In addition, this form of $l$ is not consistent with the expected scaling near the surface where $l \propto z$ (Brost and Wyngaard, 1978). The presence of the ground makes the problem for determining a suitable length scale more complicated.

Backscatter may also be important in improving a LES in order to simulate the very stable boundary layer. Currently, one of the highest stably stratified boundary layers successfully studied using LES was performed by Brown et al. (1994) where the stability parameter $\frac{h}{L}=4-5$. Earlier simulations of the same case without backscatter (Mason and Derbyshire, 1990) resulted in the development of unusual kinks in the total heat flux profiles in a manner similar to the kinks that were observed in the current study when the boundary layer was cooled too quickly or when larger negative values of $\langle w \theta\rangle_{o}$ were applied. Their SGS model was also Smagorinsky based, using stability corrected coefficients akin to the application of $l$ in the current study. With the inclusion of backscatter in their SGS model they were able to overcome the above problems (Brown et al., 1994), however their boundary layer was still considered to be weakly stable and the highly intermittent regime was not reached.

These issues and questions need to be addressed in order to move forward. An improved version of the current SGS model or an altogether new one most likely will be needed in order to perform LES of the very stable boundary layer. We believe that recent work in the development of other SGS models and new field campaigns with state of the art instrumentation can help us develop a better SGS model for the stable boundary layer. 


\section{Acknowledgements}

We thank Don Lenschow and Jeff Weil for constructive comments on this manuscript. We also wish to thank the anonymous reviewers whose comments improved the presentation of this work. EMS gratefully acknowledges the Advanced Studies Program at NCAR for support during the course of this work.

\section{Appendix A}

This section briefly outlines the methodology used to determine the eigenvalues of the Taylor-Goldstein presented in Equation (23). This equation can be rewritten as,

$$
\begin{gathered}
C^{2}\left(W^{\prime \prime}-k^{2} W\right)+C\left(-2 U W^{\prime \prime}+U^{\prime \prime} W+2 U k^{2} W\right) \\
+\left(U^{2} W^{\prime \prime}+N^{2}-U U^{\prime \prime} W-k^{2} U^{2} W\right)=0 .
\end{gathered}
$$

This is a nonlinear eigenvalue problem for the complex wave speed, $C=c+i c_{i}$, with boundary conditions, $W=0$ at $z=0$ and $z=L_{z}$. To solve this problem, we use the companion matrix method by Bridges and Morris (1984) that reformulates this nonlinear equation for $C$ into a linear form.

After discretizing Equation (A1) in the $z$ direction over $N_{z}$ points using second order finite differences, the resulting system of equations can be written in matrix form, i.e.,

$$
\left(\mathbf{A} C^{2}+\mathbf{B} C+\mathbf{D}\right) \mathbf{W}=\mathbf{0} .
$$

Here, $\mathbf{W}$ represents the a vector of unknowns, while $\mathbf{A}, \mathbf{B}$, and $\mathbf{D}$ are matrices

$$
\begin{aligned}
& \mathbf{A}=\boldsymbol{\delta}_{z}-k^{2} \mathbf{I} \\
& \mathbf{B}=\left(-2 U \boldsymbol{\delta}_{z}\right)+\left(U^{\prime \prime}+2 U k^{2}\right) \mathbf{I} \\
& \mathbf{C}=\left(U^{2} \boldsymbol{\delta}_{z}\right)+\left(N^{2}-U U^{\prime \prime}-k^{2} U^{2}\right) \mathbf{I}
\end{aligned}
$$

where $\boldsymbol{\delta}_{z}$ is the matrix of finite difference coefficients and $\mathbf{I}$ is the identity matrix.

Following Bridges and Morris (1984), we can rewrite this system once again in the generalized eigenvalue form of

$$
\left(\begin{array}{cc}
-\mathbf{B} & -\mathbf{D} \\
\mathbf{I} & 0
\end{array}|-C| \begin{array}{cc}
\mathbf{A} & 0 \\
0 & \mathbf{I}
\end{array} \mid\right)\left(\begin{array}{c}
C \mathbf{W} \\
\mathbf{W}
\end{array}\right)=0 .
$$

Note that since the boundary conditions are zero, we only solve for the interior points such that the matrices in Equation (A4) are not singular. The eigenvalue problem is then solved using a standard eigenvalue software package. The solution 
method and code were tested by reproducing the results of Hazel (1972) for the case studying the stability of the hyperbolic tangent shear layer.

\section{References}

Andren, A.: 1995, 'The Structure of Stably Stratified Atmospheric Boundary Layers: A Large-Eddy Simulation Study', Quart. J. Roy. Meteorol. Soc. 121, 961-985.

Blackadar, A. K.: 1957, 'Boundary Layer Wind Maxima and their Significance for the Growth of Nocturnal Inversions', Bull. Amer. Meteorol. Soc. 38, 283-290.

Bridges, T. J. and Morris, P. J.: 1984, 'Differential Eigenvalue Problems in which the Parameter Appears Nonlinearly', J. Comp. Phys. 55, 437-460.

Brost, R. A. and Wyngaard, J. C.: 1978, 'A Model Study of the Stably Stratified Planetary Boundary Layer', J. Atmos. Sci. 35, 1427-1440.

Brown, A. R., Derbyshire, S. H., and Mason, P. J.: 1994, 'Large-Eddy Simulation of Stable Atmospheric Boundary Layers with a Revised Stochastic Subgrid Model', Quart. J. Roy. Meteorol. Soc. 120, 1485-1512.

Businger, J. A., Wyngaard, J. C., Izumi, Y., and Bradley, E. F.: 1971, 'Flux-Profile Relationships in the Atmospheric Surface Layer', J. Atmos. Sci. 28, 181-189.

Carruthers, D. J. and Moeng, C.-H.: 1987, 'Waves in the Overlying Inversion of the Convective Boundary Layer', J. Atmos. Sci. 44, 1801-1808.

Coleman, G. N., Ferziger, J. H., and Spalart, P. R.: 1992, 'Direct Simulation of the Stably Stratified Turbulent Ekman Layer', J. Fluid Mech. 244, 677-712.

Deardorff, J. W.: 1980, 'Stratocumulus-Capped Mixed Layers Derived from a Three-Dimensional Model', Boundary-Layer Meteorol. 18, 495-527.

Delage, Y.: 1974, 'A Numerical Study of the Nocturnal Atmospheric Boundary Layer', Quart. J. Roy. Meteorol. Soc. 100, 351-364.

Derbyshire, S. H.: 1990, 'Nieuwstadt's Stable Boundary Layer Revisited', Quart. J. Roy. Meteorol. Soc. 116, 127-158.

Derbyshire, S. H.: 1995, 'Stable Boundary Layers: Observations, Models and Variability. Part I: Modelling and Measurements', Boundary-Layer Meteorol. 74, 19-54.

Finnigan, J. J., Einaudi, E., and Fua, D.: 1984, 'The Interaction between an Internal Gravity Wave and Turbulence in the Stably-Stratified Nocturnal Boundary Layer', J. Atmos. Sci. 41, 2409-2436.

Galmarini, S., Beets, C., Duynkerke, P. G., and Vilã-Guerau de Arellano, J.: 1988, 'Stable Nocturnal Boundary Layers: A Comparison of One-Dimensional and Large-Eddy Simulation Models', Boundary-Layer Meteorol. 88, 181-210.

Gossard, E. E. and Hooke, W. H.: 1975, Waves in the Atmosphere, Elsevier Scientific Publishing Company, Amsterdam, 456 pp.

Hazel, P.: 1972, 'Numerical Studies of the Stability of Inviscid Stratified Shear Flows', J. Fluid Mech. 51, 39-61.

Hunt, J. C. R., Kaimal, J. C., and Gaynor, J. E.: 1985, 'Some Observations of Turbulence Structure in Stable Layers', Quart. J. Roy. Meteorol. Soc. 111, 783-815.

Klemp, J. B. and Durran, D. R.: 1983, 'An Upper Boundary Condition Permitting Internal Gravity Wave Radiation in Numerical Mesoscale Models', Mon. Wea. Rev. 111, 430-444.

Kosovic, B. and Curry, J. A.: 2000, 'A Large-Eddy Simulation Study of a Quasi-Steady StablyStratified Atmospheric Boundary Layer', J. Atmos. Sci., in press.

Lin, C.-L., McWilliams, J. W., Moeng, C.-H., and Sullivan, P. P.: 1996, 'Coherent Structures and Dynamics in a Neutrally Stratified Planetary Boundary Layer Flow’, Phys. Fluids 8, 2626-2639.

Mahrt, L.: 1998, 'Stratified Atmospheric Boundary Layers and Breakdown of Models', Theor. Comp. Fluid Dyn. 11, 263-280. 
Mason, P. J. and Derbyshire, S. H.: 1990, 'Large-Eddy Simulation of the Stably-Stratified Atmospheric Boundary Layer', Boundary-Layer Meteorol. 53, 117-162.

Moeng, C.-H.: 1984, 'A Large-Eddy-Simulation Model for the Study of Planetary Boundary-Layer Turbulence', J. Atmos. Sci. 2052-2062.

Moeng, C.-H. and Sullivan, P. P.: 1994, 'A Comparison of Shear- and Buoyancy-Driven Planetary Boundary Layer Flows', J. Atmos. Sci. 51, 999-1022.

Nieuwstadt, F. T. M.: 1984, 'The Turbulent Structure of the Stable, Nocturnal Boundary Layer', J. Atmos. Sci. 41, 2202-2216.

Stull, R. B.: 1988, An Introduction to Boundary Layer Meteorology, Kluwer Academic Publishers, Dordrecht, $666 \mathrm{pp}$.

Sullivan, P. P., McWilliams, J. C., and Moeng,, C.-H.: 1994, 'A Subgrid-Scale Model for Large-Eddy Simulation of Planetary Boundary-Layer Flows', Boundary-Layer Meteorol. 71, 247-276.

Sullivan, P. P., McWilliams, J. C., and Moeng, C.-H.: 1996, 'A Grid Nesting Method for Large-Eddy Simulation of Planetary Boundary-Layer Flows', Boundary-Layer Meteorol. 80, 167-202. 